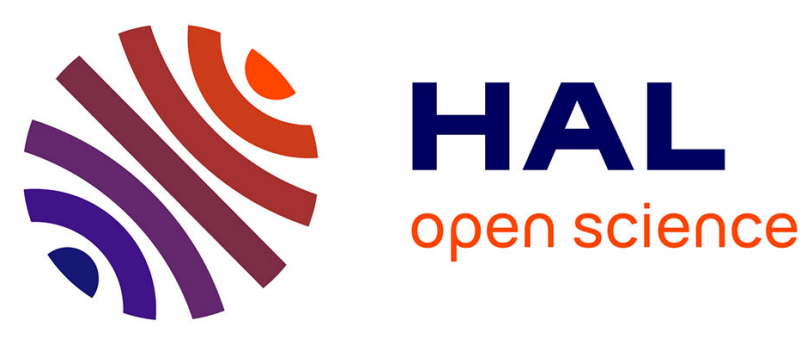

\title{
Evolution morpho-sédimentaire et enregistrement pollinique atlantique dans le nord de la France: la séquence de Valenciennes "le vignoble" (vallée de l'Escaut, nord)
}

Muriel Boulen, Laurent Deschodt, Alain Henton

\section{To cite this version:}

Muriel Boulen, Laurent Deschodt, Alain Henton. Evolution morpho-sédimentaire et enregistrement pollinique atlantique dans le nord de la France: la séquence de Valenciennes "le vignoble" (vallée de l'Escaut, nord). Quaternaire, 2014, 25 (4), pp.369-389. 10.4000/quaternaire.7290 . hal-01396969

\section{HAL Id: hal-01396969 \\ https://hal.science/hal-01396969}

Submitted on 15 Nov 2016

HAL is a multi-disciplinary open access archive for the deposit and dissemination of scientific research documents, whether they are published or not. The documents may come from teaching and research institutions in France or abroad, or from public or private research centers.
L'archive ouverte pluridisciplinaire HAL, est destinée au dépôt et à la diffusion de documents scientifiques de niveau recherche, publiés ou non, émanant des établissements d'enseignement et de recherche français ou étrangers, des laboratoires publics ou privés. 


\title{
ÉVOLUTION MORPHO-SÉDIMENTAIRE ET ENREGISTREMENT POLLINIQUE ATLANTIQUE DANS LE NORD DE LA FRANCE : LA SÉQUENCE DE VALENCIENNES « LE VIGNOBLE » (VALLÉE DE L'ESCAUT, NORD)
}

\author{
Muriel BOULEN ${ }^{1}$, Laurent DESCHODT ${ }^{2} \&$ Alain HENTON ${ }^{3}$
}

\begin{abstract}
RÉSUMÉ
Le site du Vignoble occupe la jonction entre le fond de vallée de l'Escaut et un talweg descendant du versant. Un cône de déjection pléistocène a développé une petite zone basse en périphérie. Elle est comblée par un limon colluvié attribué au début de l'Holocène, puis par 1,5 m de tourbe formée durant l'Atlantique. L'enregistrement bénéficie de sept dates radiocarbone, comprises entre $7310 \pm 59 \mathrm{BP}$ (soit environ 8100 ans cal. BP) et $5422 \pm 43 \mathrm{BP}$ (soit environ 6300 cal. BP), et d'une étude palynologique. Au cours de l'Atlantique, le diagramme pollinique présente un enregistrement récurrent de rudérales, parfois associé à un recul des essences forestières. Cependant, l'analyse permet d'exclure une causalité anthropique automatique. En revanche, différentes phases de recul de la chênaie semblent être liées à des variations des conditions édaphiques. La dernière phase humide, la plus importante, débute au sommet de la tourbe et fait suite aux premiers indices d'agriculture néolithique.
\end{abstract}

Mots-clés : Escaut (nord de la France), Holocène, Atlantique, palynologie, tourbe, variations d'humidité, anthropisation

\section{ABSTRACT}

MORPHO-SEDIMENTARY EVOLUTION AND PALYNOLOGICAL RECORD OF ATLANTIC PHASE IN NORTHERN FRANCE: THE SEQUENCE OF VALENCIENNES “LE VIGNOBLE” (SCHELDE VALLEY, NORTH DEPARTEMENT)

The Vignoble site is located at the junction between the bottom of the Schelde valley and a talweg coming from the slope. A Pleistocene alluvial fan created a peripheral small low area. This is filled in by a silt colluvium attributed to the early Holocene, and then by about $1.5 \mathrm{~m}$ of peat during the Atlantic. The record is constrained by seven radiocarbon dates, between 7,310 $\pm 59 \mathrm{BP}$ (ca. 8,100 cal. yr BP) and 5,422 $\pm 43 \mathrm{BP}$ (ca. 6,300 cal. yr BP) and a palynological study. During the Atlantic palynological data show a recurrent record of ruderal species, occasionally associated with a regression of forest trees. However, the analysis allows to exclude systematic anthropic causality for the evolution of the vegetation. On the contrary, different phases of declining oak appear to be related to changes in soil conditions. The last and strongest wet phase began at the upper surface of the peat, following the first indications of Neolithic agriculture.

Keywords: Schelde River (northern France), Holocene, Atlantic, palynology, peat, humidity variations, anthropisation

\section{INTRODUCTION}

Si les séquences polliniques qui couvrent l'Atlantique commencent à être plutôt bien documentées au sein du Bassin parisien (Leroyer et al., 2013) et en Belgique (Munaut, 1967), de tels enregistrements polliniques, associés à une étude chrono-stratigraphique sont encore anecdotiques sur le nord de la France. Cette chronozone est définie par certaines caractéristiques communes, mais des différences régionales apparaissent néanmoins dans la composition du couvert végétal (Leroyer et al., 2013). Caractérisée par une phase d'optimum climatique (Magny, 1995), elle présente néanmoins des phases d'intensification des anomalies et des changements rapides des conditions climatiques (Gronenborn, 2010).

La séquence tourbeuse atlantique de Valenciennes « Le Vignoble » a fait l'objet d'une étude litho-stratigraphique, associée à une analyse palynologique. Ces données permettent de proposer l'histoire du vallon et

\footnotetext{
${ }^{1}$ INRAP/UMR 7209. Centre de Recherches Archéologiques, Abbaye Saint-Jean des Vignes, FR-02200 SOISSONS.

Courriel : muriel.boulen@inrap.fr

${ }^{2}$ INRAP/UMR 8591. Centre de Recherches Archéologiques, ZI de la Pilaterie, 11 rue des champs, FR-59350 VILLENEUVE-D’ASCQ. Courriel : laurent.deschodt@inrap.fr

${ }^{3}$ INRAP/Halma-Ipel UMR 8164. Centre de Recherches Archéologiques, ZI de la Pilaterie, 11 rue des champs, FR-59350 VILLENEUVE-

D’ASCQ. Courriel : alain.henton@inrap.fr
} 
de l'évolution de la couverture végétale, mettant ainsi en évidence des variations des conditions édaphiques entre environ 8200 et 5000 ans cal. BP. Ces résultats sont alors confrontés aux données régionales et extrarégionales.

\section{2 - CADRE DE L'́́TUDE}

\section{1 - CONTEXTE}

La ville de Valenciennes, dans le nord de la France, occupe la bordure septentrionale du Bassin parisien, à la limite des affleurements des assises crayeuses et marneuses crétacées. Le relief de plateau s'y estompe et la vallée de l'Escaut entre dans un relief de plaine (fig. 1). Un peu en amont de la ville, le lieu-dit « Le Vignoble » est un versant d'exposition est qui domine une ancienne gravière (l'« Étang du Vignoble »). Sur prescription du Service Régional de l'Archéologie (indices d'une occupation néolithique), une fouille archéologique a été entreprise sur l'emplacement d'un futur bassin de rétention d'eau (Henton et al., 2009), au contact du fond de vallée et du versant. À cet endroit, un talweg descend du plateau et rejoint le fond de vallée.

\section{2 - DATATIONS}

Une étude dendrochronologique et huit datations radiocarbone ont été réalisées (tab. 1). Sept d'entre elles ont été faites sur la tourbe, et une datation a été effectuée sur les cernes externes d'un chêne daté par ailleurs par dendrochronologie (Dendrotech, rapport DT-09-014). La confrontation des datations permet de conclure à la chute de l'arbre dans la zone humide au cours de l'Atlantique et son enfouissement progressif avec la croissance de la tourbière. Une série de 268 cernes consécutifs a été acquise. En l'absence de référentiels locaux sur la période donnée par les datations radiocarbone, la séquence a été testée sur les référentiels néolithiques allemands. Les résultats des calculs, qui sont visuellement et statistiquement très acceptables, positionnent le dernier cerne mesuré (sans aubier $=$ terminus post quem) en 4930 av. J.-C., soit entre 4985 et 4829 ans av. J.-C., âge calibré avec un intervalle d'erreur de 1 sigma.

\section{3 - TECHNIQUES ET MÉTHODES}

Un décaissement à la pelle mécanique, sur une profondeur maximale de près de $4 \mathrm{~m}$, a permis le levé de coupes en paliers (coupes 1 et 2), complétées par une tranchée en fond de décapage (coupe 3) (fig. 1). Les trois coupes permettent d'esquisser une synthèse lithostratigraphique (voir la figure 3 et le tableau 2 pour les observations détaillées, et la figure 4 et le tableau 2 pour la synthèse) et ont fait l'objet de prélèvements palynologiques. Les différents prélèvements représentent une séquence qui se développe sur près de $3,5 \mathrm{~m}$ (fig. 2), dont la partie supérieure est constituée de sédiments organiques sur
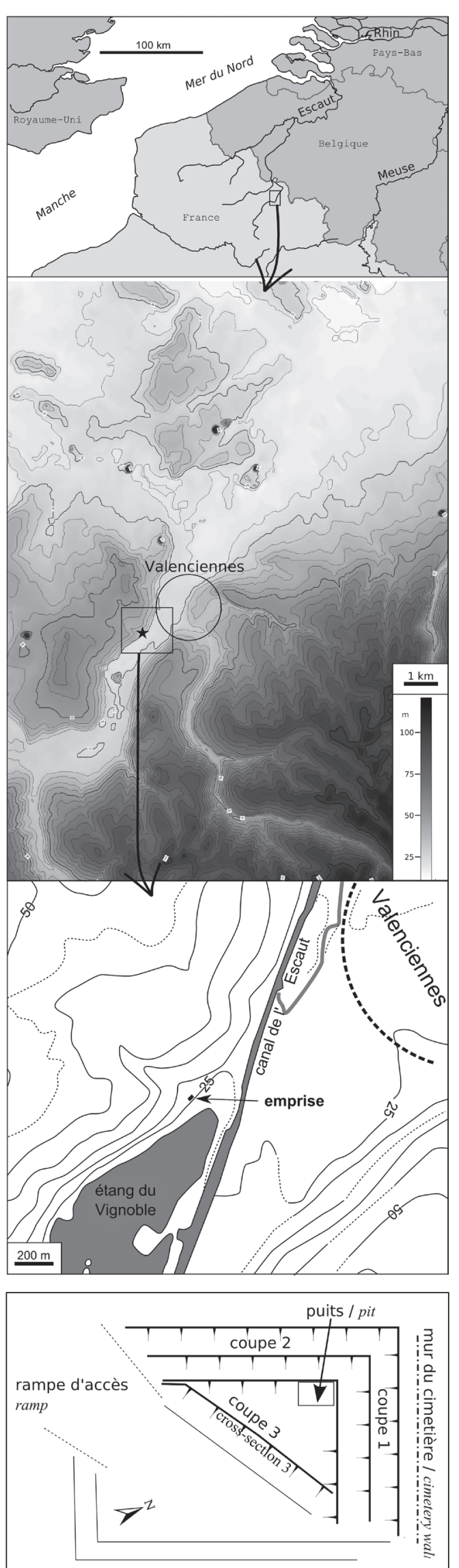

Fig. 1 : Localisation du site de Valenciennes « le Vignoble » dans la vallée de l'Escaut et localisation des coupes.

Courbes de niveau en mètres NGF Lallemand, équidistance de $5 \mathrm{~m}$, d'après la carte IGN au $1 / 25000$.

Fig. 1: Location of the Valenciennes "le Vignoble" site in the Schelde valley and location of the cross-sections. Contour levels in meters NGF Lallemand survey, at $5 \mathrm{~m}$ intervals, after the IGN 1/25000 map. 


\begin{tabular}{|l|l|l|l|l|}
\hline num & matériel & code & dates BP & cal. BC, 1 sigma \\
\hline D1 & tourbe & Erl-11736 & $7310 \pm 59$ & $6226-6098 \mathrm{BC}$ \\
\hline D2 & tourbe & Erl-11735 & $7043 \pm 63$ & $5998-5849 \mathrm{BC}$ \\
\hline D3 & tourbe & Erl-11734 & $6901 \pm 49$ & $5838-5732 \mathrm{BC}$ \\
\hline D4 & tourbe & Erl-11733 & $6297 \pm 43$ & $5659-5564 \mathrm{BC}$ \\
\hline D5 & tourbe & Erl-11732 & $6247 \pm 43$ & $5307-5083 \mathrm{BC}$ \\
\hline D6 & tourbe & Erl-11731 & $6076 \pm 43$ & $5053-4915 \mathrm{BC}$ \\
\hline D7 & tronc, cernes externes & Erl-11729 & $6026 \pm 42$ & $4985-4849 \mathrm{BC}$ \\
\hline D8 & tourbe & Erl-11730 & $5422 \pm 43$ & $4335-4257 \mathrm{BC}$ \\
\hline
\end{tabular}

Tab. 1 : Datations (C14-Labor, Erlangen, Allemagne).

Tab. 1: Dates (C14-Labor, Erlangen, Germany).

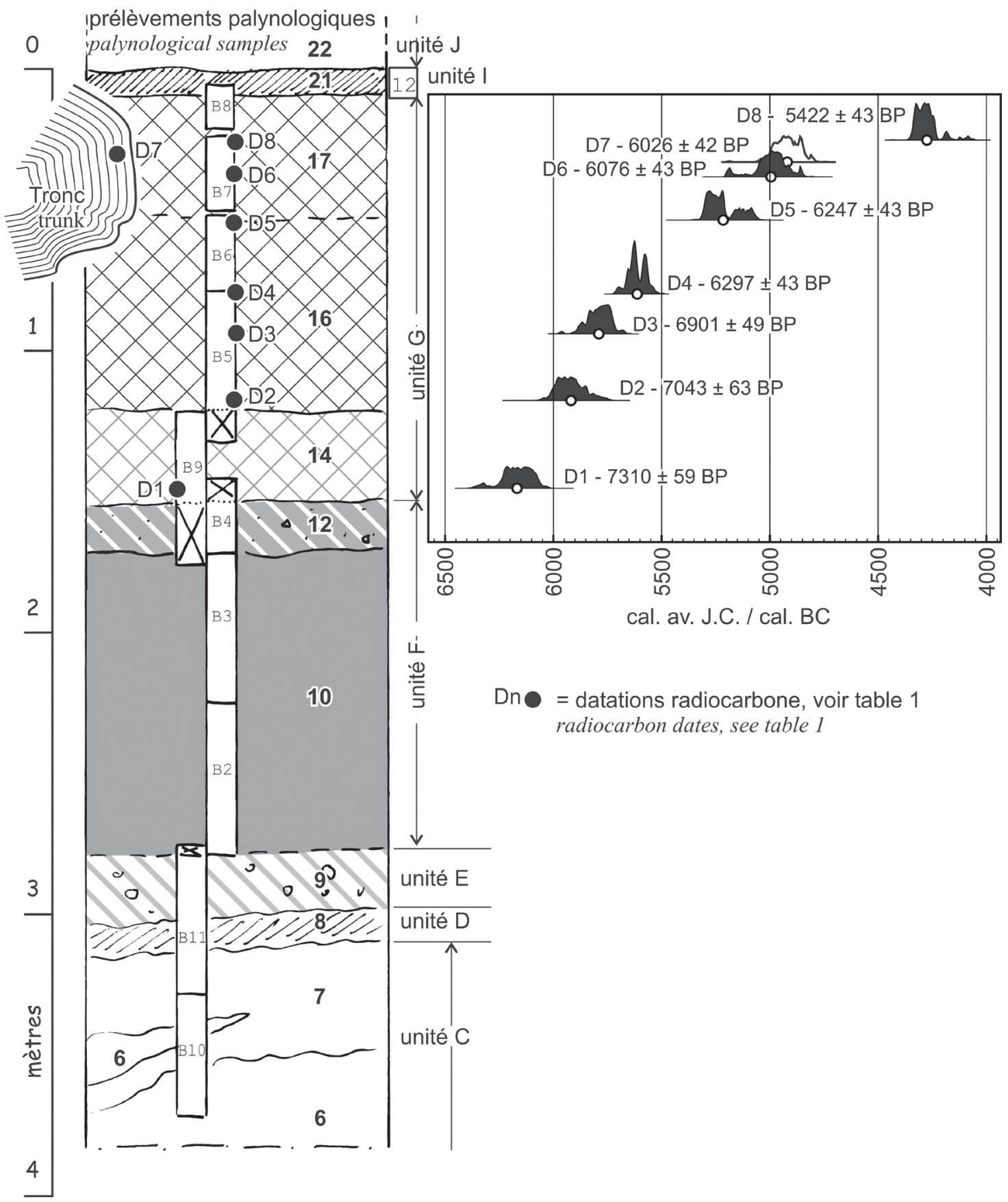

Fig. 2 : Log synthétique des prélèvements palynologiques et dates radiocarbone.

Courbe de calibration : IntCal 04 (Reimer et al., 2004).

Fig. 2: Synthetic log with palynological samples and radiocarbon dates. Calibrationcurve: IntCal 04 (Reimer et al., 2004). 
Ouest

\section{Coupe 1}

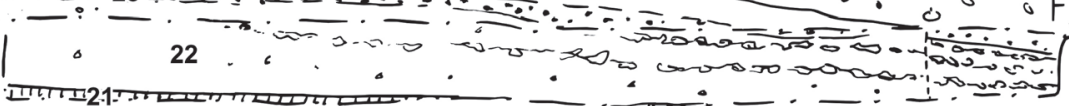

$0 \quad 1$

Sud
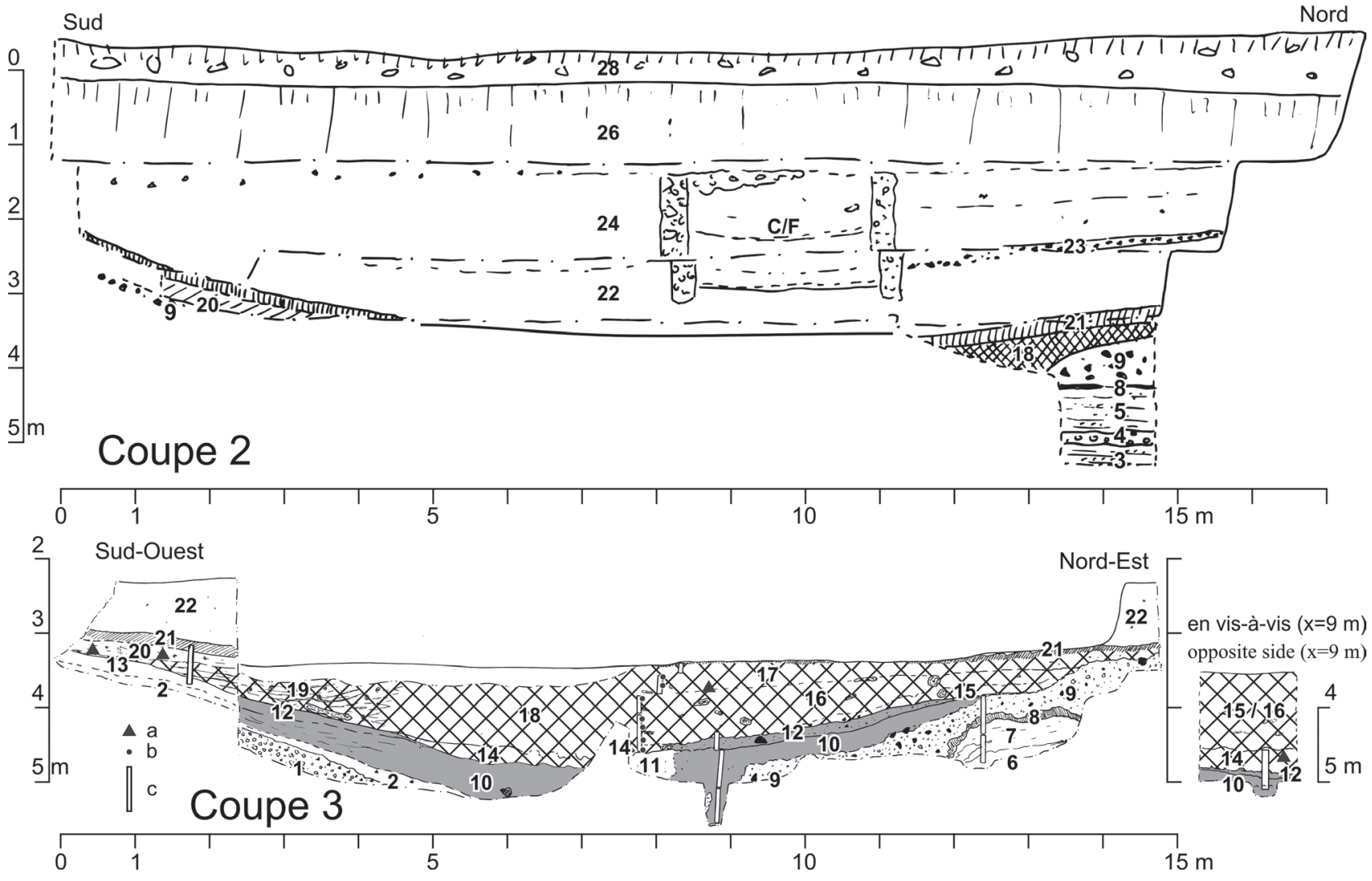

Fig. 3 : Coupes lithostratigraphiques.

Fig. 3: Lithostratigraphic cross-sections

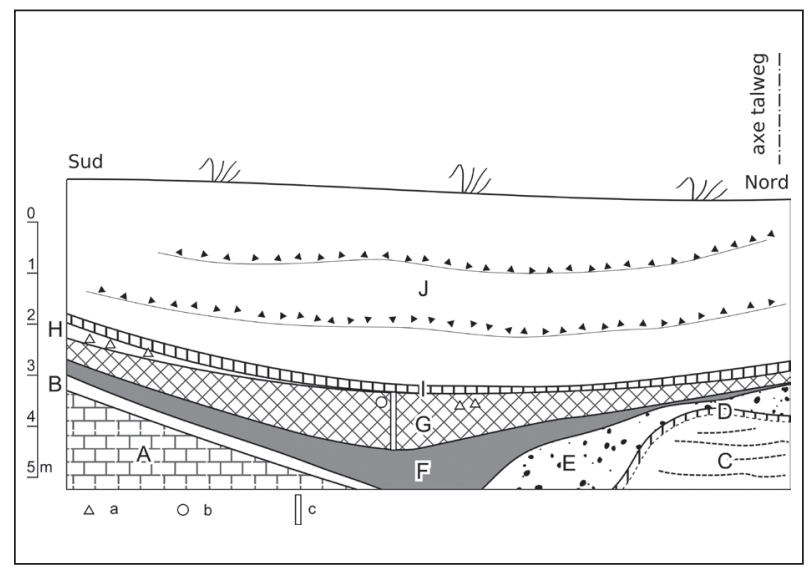

Fig. 4 : Synthèse stratigraphique.

Fig. 4: Stratigraphic synthesis.
1,5 m. L'échantillonnage a été réalisé avec une maille de $5 \mathrm{~cm}$. L'extraction du matériel sporo-pollinique suit un protocole standard (Fægri \& Iversen, 1989) utilisant une liqueur lourde de chlorure de zinc pour les sédiments minéraux, et réduit à la méthode $\mathrm{NaOH}$ pour les tourbes.

Pour qu'un échantillon donné soit interprétable, il faut qu'au minimum 200 à 300 grains de pollen aient été décomptés pour une vingtaine de taxons identifiés. Au-delà du seuil de 300 pollens, on peut considérer que l'on ne gagne plus d'information (Reille, 1990). Cependant, la lecture a systématiquement été poursuivie jusqu'à obtenir 200 grains, abstraction faite du ou des taxons prédominants. Seuls les résultats polliniques des échantillons représentatifs, soit un total de 31 répartis sur les niveaux organiques supérieurs, sont représentés dans la figure 5. Pour chacun, la somme de base utilisée 


\begin{tabular}{|c|c|c|c|c|c|}
\hline $\begin{array}{l}\text { Synthèse (fig. 4) } \\
\text { synthesis (fig. 4) }\end{array}$ & interprétation & $\begin{array}{c}\text { coupe } \\
1\end{array}$ & \begin{tabular}{c|c|} 
coupe \\
2
\end{tabular} & $\begin{array}{c}\text { coupe } \\
3\end{array}$ & détail (fig. 3) / details (fig. 3) \\
\hline $\begin{array}{l}\text { A - Craie fragmentée / } \\
\text { chalk fragments }\end{array}$ & & & & 1 & craie fragmentée / fragmented chalk \\
\hline $\begin{array}{l}\text { B - Limon homogène } \\
\text { gleyifié / homogeneous } \\
\text { gleyified loam }\end{array}$ & $\begin{array}{l}\text { Pléistocène, formation de } \\
\text { versant } \\
\text { Pleistocene, slope formation }\end{array}$ & & & 2 & limon verdâtre plastique / plastic greenish loam \\
\hline \multirow{5}{*}{$\begin{array}{l}\text { C - Limon lité sablo- } \\
\text { graveleux / bedded } \\
\text { sandy and gravelly loam }\end{array}$} & \multirow{5}{*}{$\begin{array}{c}\text { Pléistocène, alluvions de fond } \\
\text { de vallée et/ou cône de } \\
\text { déjection } \\
\text { Pleistocene, bottom balley } \\
\text { alluvium and/or alluvial fan }\end{array}$} & & 3 & & limon et sable lité, bleuté / bluish bedded sand and loam \\
\hline & & & 4 & & sable, gravier de craie, silex / sand, chalk gravels, flints \\
\hline & & & 5 & & $\begin{array}{l}\text { lits de limon et sable, gley (bleu) sauf en tête ou passage très } \\
\text { clair, calcaire / sand and loam beds, gley (blue) except at top } \\
\text { where calcareous very light part }\end{array}$ \\
\hline & & & & 6 & $\begin{array}{l}\text { limon jaune orangé sableux avec lits calcaires / orangey } \\
\text { yellow sandy loam with calcareous beds }\end{array}$ \\
\hline & & & & 7 & $\begin{array}{l}\text { limon blanc, lité, très calcaire / white loam, bedded, very } \\
\text { calcareous }\end{array}$ \\
\hline D - Sol noir / black soil & $\begin{array}{l}\text { pédogenèse interstadiaire } \\
\text { Tardiglaciaire, Allerød? } \\
\text { lateglacial interstadial } \\
\text { pedogenesis, Allerød? }\end{array}$ & & 8 & 8 & limon plastique gris noir / grey black plastic loam \\
\hline $\begin{array}{l}\text { E - Silex (matrice } \\
\text { limoneuse) / flint (silty } \\
\text { matrix) }\end{array}$ & $\begin{array}{c}\text { dépôt grossier au pied du } \\
\text { versant, fluviatile de versant } \\
\text { Dryas récent? } \\
\text { coarse deposit at slope foot, } \\
\text { slope fluviatil, Younger Dryas } \\
\text { ? }\end{array}$ & & 9 & 9 & $\begin{array}{l}\text { limon gris sombre verdâtre, très sableux, plastique, très } \\
\text { chargé en silex, certains de tailles pluri-centimétriques, } \\
\text { hétérogène; passages sableux dans la coupe } 2 \text { / greenish } \\
\text { dark grey loam, very sandy, plastic, with numerous flints, } \\
\text { which are few centimeters sized, heterogeneous ; sandy beds } \\
\text { in cross-section } 2\end{array}$ \\
\hline \multirow{4}{*}{$\begin{array}{l}\mathrm{F} \text { - Sable et limon } \\
\text { plastique / sand and } \\
\text { plastic loam }\end{array}$} & \multirow{4}{*}{$\begin{array}{l}\text { colluvion en pied de versant, } \\
\text { début Holocène } \\
\text { slope foot colluviums, early } \\
\text { Holocene }\end{array}$} & & & 10 & limon gris sombre, plastique / plastic dark grey loam \\
\hline & & & & 11 & $\begin{array}{l}\text { id. 9, un peu moins riche en éléments grossiers / idem 9, less } \\
\text { coarse elements }\end{array}$ \\
\hline & & & & 12 & $\begin{array}{l}\text { sable clair bleuté, quelques éléments de gravier de craie et de } \\
\text { silex / bluish light sand, few chalk and flint gravels }\end{array}$ \\
\hline & & & & 13 & $\begin{array}{l}\text { limon clair (transition latérale de } 12 \text { ?) / light loam (12 lateral } \\
\text { transition?) }\end{array}$ \\
\hline \multirow{6}{*}{ G - Tourbe / peat } & \multirow{6}{*}{$\begin{array}{c}\text { Envahissement tourbeux } \\
\text { provenant du fond de vallée }+ \\
\text { écoulements sporadiques } \\
\text { provenant du versant, } \\
\text { Atlantique } \\
\text { Peat infilling coming from the } \\
\text { valley bottom + sporadic } \\
\text { flows coming from the slope, } \\
\text { Atlantic }\end{array}$} & & & 14 & $\begin{array}{l}\text { limon tourbeux gris brun, plastique ; plus épais vers l'Escaut } \\
\text { et limite inférieure très irrégulière (figures de charge ou } \\
\text { piétinement?) ; présence de silex taillé } \\
\text { brown grey peaty loam, plastic ; thicker towards the Schelde } \\
\text { and irregular bottom limit (loadcast or tramping?) ; knapped } \\
\text { flints }\end{array}$ \\
\hline & & & & 15 & $\begin{array}{l}\text { tourbe limoneuse, agrégats provenant des unités sous- } \\
\text { jacentes / loamy pead, agregats coming from underlaying } \\
\text { units }\end{array}$ \\
\hline & & & & 16 & $\begin{array}{l}\text { tourbe franche fibreuse un peu rousse, légèrement sableuse } \\
\text { (quelques lits sableux) vers le nord, avec bois, noisettes / } \\
\text { reddish fibrous pead with some moss, lightly sandy (few } \\
\text { sandy beds) northwards, with wood, nuts }\end{array}$ \\
\hline & & & & 17 & tourbe fibreuse très compacte / very compact fibrous peat \\
\hline & & & 18 & 18 & tourbe fibreuse, indifférenciée / fibrous pead \\
\hline & & & & 19 & $\begin{array}{l}\text { passe à tourbe calcaire, finement litée, avec malacofaune / } \\
\text { calcareous peat, fine beds, malacofauna }\end{array}$ \\
\hline $\begin{array}{l}\mathrm{H} \text { - Limon gris / grey } \\
\text { loam }\end{array}$ & $\begin{array}{l}\text { colluvions, Atlantique ou } \\
\text { postérieur } \\
\text { colluviums, Atlantic or later }\end{array}$ & & 20 & 20 & $\begin{array}{l}\text { limon gris, charbon de bois, industrie lithique / grey loma, } \\
\text { charcoal, knapped flints }\end{array}$ \\
\hline I - Sol noir / black soil & $\begin{array}{l}\text { sol de marais subatlantique / } \\
\text { subatlantic swamp soil }\end{array}$ & 21 & 21 & 21 & $\begin{array}{l}\text { limon plastique noir, peu compact, " mou ", limite inférieure } \\
\text { diffuse; présence de tessons de céramiques du Haut Moyen } \\
\text { Age / black plastic loam, loosely, soft, diffuse bottom limit ; } \\
\text { Early Middle Ages ceramics }\end{array}$ \\
\hline \multirow{8}{*}{$\begin{array}{l}\text { J - Limon, silex, } \\
\text { structures / loam, flints, } \\
\text { structures }\end{array}$} & \multirow{8}{*}{$\begin{array}{l}\text { colluvions et remaniements } \\
\text { anthropiques récents } \\
\text { colluviums and recent } \\
\text { anthropic reworking }\end{array}$} & 22 & 22 & 22 & $\begin{array}{l}\text { limon brun peu compact, humide, tendance à l'éboulement, } \\
\text { avec cailloutis de silex épars ; plusieurs lignes bien distinctes } \\
\text { de « mottes" remaniées de limon poudreux très clair avec } \\
\text { lamines plus sombres. Interprétation: labour de croûtes de } \\
\text { battance, contexte agricole et colluvial en bas de pente / } \\
\text { loosely brow loam, damp, collapse tendancy, scarse flint } \\
\text { gravels; severals bands of reworked dark and light dusty } \\
\text { loam laminae blocks. Interpretation : slaking crusts ploughing, } \\
\text { foot slope colluvium in an agricultural context }\end{array}$ \\
\hline & & 23 & 23 & & $\begin{array}{l}\text { limon brun très chargé en cailloutis (silex); cailloutis très } \\
\text { dense vers l'est / brown loam rich in gravels (flint); very } \\
\text { dense gravels eastwards }\end{array}$ \\
\hline & & 24 & 24 & & $\begin{array}{l}\text { limon brun gris, nombreux cailloutis et inclusions divers } \\
\text { (fragments brique, craie...) / grey brown loam, numerous } \\
\text { peebles and various inclusions (brick fragments, chalk } \\
\text { gravels...) }\end{array}$ \\
\hline & & 25 & & & brique, craie, remblais / brick, chalk, embankment \\
\hline & & 26 & 26 & & $\begin{array}{l}\text { limon brun sombre, humifère, avec nombreux éléments } \\
\text { anthropiques / dark brown loam, humic, with numerous } \\
\text { antropic elements }\end{array}$ \\
\hline & & 27 & & & schiste minier, scories.../ pelite, scoria \\
\hline & & 28 & 28 & & $\begin{array}{l}\text { horizon humifère développé sur des remblais (briques...) / } \\
\text { humic horizon upon embankment (bricks...) }\end{array}$ \\
\hline & & $\mathrm{C} / \mathrm{F}$ & $\mathrm{C} / \mathrm{F}$ & & $\begin{array}{l}\text { caves, fosses et fondations modernes ou contemporaines, } \\
\text { briques, béton... / cellars, pits and modern or contemporary } \\
\text { fondations, brick, concrete }\end{array}$ \\
\hline
\end{tabular}

Tab. 2 : Synthèse stratigraphique.

Tab. 2: Stratigraphic synthesis. 


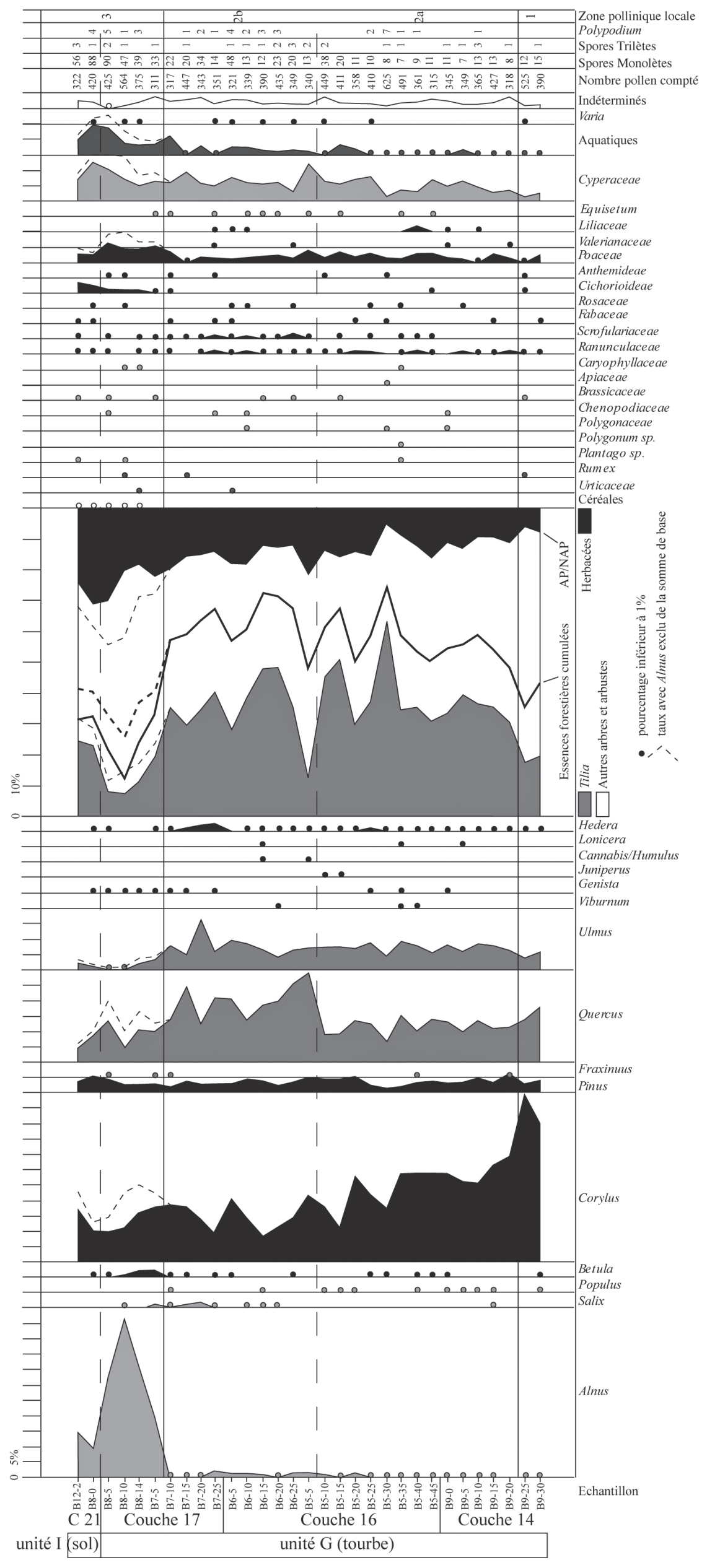

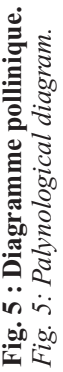


pour le calcul des pourcentages prend en compte le total du pollen des arbres et arbustes (AP $=$ arborean pollen / pollen arboré) et des herbacées (NAP $=$ non arborean pollen / pollen non arboré). Le rapport AP/NAP représente donc la part des grains de pollen d'arbres et arbustes sur ceux de la strate herbacée. Les spores sont sorties de la somme de base.

Les différentes plantes aquatiques ont été regroupées (détail par échantillon dans le tableau 3) ; il s'agit de Potamogeton (potamot), Sagittaria (sagittaire), Typha latifolia (massette à larges feuilles), T. minima (petite massette) et T. angustifolia (massette à feuilles étroites), Sparganium (rubanier), Callitriche (callitriche), Hydrocharis (hydrocharis) et Lemna (lentille d'eau) (Blamey \& Grey-Wilson, 1991).

Quelques taxons herbacés, présents de manière sporadique, sont représentés en colonne «varia» : B8-0 : Veronica ; B8-10 : Sedum type, Rubiaceae ; B8-14 : Convolvulaceae ; B7-25 : Sedum type ; B6-5 : Lamiaceae, Saxifragaceae ; B6-15 : Ericaceae ; B6-25 : Sedum type ; B5-10 : Ericaceae ; B5-25 : Lamiaceae ; B9-25 : Galium type.
Par ailleurs, les courbes des principaux taxons ou groupements ont également été construites avec Tilia (tilleul) exclu de la somme de base (fig. 6).

Enfin, pour aider à l'interprétation des diverses variations dans les courbes polliniques, et de l'impact des unes sur les autres, une figure synthétique est proposée (fig. 7).

Différentes courbes y apparaissent :

- la courbe des essences forestières cumulées, qui prend en compte Quercus (chêne), Tilia, Ulmus (orme) et Fraxinus (frêne);

- les indicateurs des conditions édaphiques, avec, d'une part, le cortège des plantes aquatiques, énumérées précédemment, et, d'autre part, les taxons qui constituent une prairie humide, avec Equisetum (les presles) et les Cyperaceae, associées aux Poaceae. Sur les 52 espèces de Cyperaceae répertoriées dans le nord de la France et la Belgique, 2 seulement poussent dans des milieux secs, 15 sont plus ubiquistes, et 35 sont inféodées à des milieux humides (Bonnier \& de Layens, 2008). Néanmoins, malgré une détermination qui se limite au rang de la famille, la communauté des palynologues considère ce taxon comme hygrophile (Marguerie, 2009 ; Macaire et al., 2010), indicateur d'une prairie humide (Richard, 1995 ; Gaudin (2004) in

\begin{tabular}{|c|c|c|c|c|c|c|c|c|c|}
\hline Echantillon & Potamogeton & Sagittaria & Typha lat. & Typha min. & Typha ang. & Sparganium & Callitriche & Hydrocharis & Lemna \\
\hline B12-2 & & & 0,3 & & 0,6 & & & & 4,0 \\
\hline B8-0 & 0,5 & & & & & & & & 9,3 \\
\hline B8-5 & 0,5 & & & & & & & & 8,2 \\
\hline B8-10 & & & & & & & & & 3,7 \\
\hline B8-14 & 0,3 & & 0,3 & & & & & & 2,7 \\
\hline B7-5 & & & & & 0,3 & & 0,3 & & 2,9 \\
\hline B7-10 & & & & & & & & & 6,0 \\
\hline B7-15 & 0,9 & & & & & & & & \\
\hline B7-20 & 0,3 & & & & & & & & 2,6 \\
\hline B7-25 & & & & & & & & & 0,3 \\
\hline B6-5 & 0,3 & & & & & & & & 2,5 \\
\hline B6-10 & 0,9 & & & & & & & & 1,5 \\
\hline B6-15 & & & 0,3 & & & & & & 1,3 \\
\hline B6-20 & & & 0,2 & & & & & & 0,9 \\
\hline B6-25 & & & 0,6 & & 0,3 & & 0,3 & & 0,6 \\
\hline B5-5 & & & & & 0,3 & 0,6 & & & 0,3 \\
\hline B5-10 & & & & & 0,2 & & & & 0,4 \\
\hline B5-15 & & 0,2 & & & & 1,2 & & & 1,7 \\
\hline B5-20 & 0,8 & & & & 0,3 & & & & 0,6 \\
\hline B5-25 & & & & & & & & & 0,2 \\
\hline B5-30 & 0,2 & 0,2 & & & & 0,2 & & & 0,2 \\
\hline B5-35 & & & & & & & & & 0,2 \\
\hline B5-40 & & & & & & & & & 0,6 \\
\hline B5-45 & & & & & 0,3 & & & & 0,6 \\
\hline B9-0 & 0,3 & & & & & & & & 0,3 \\
\hline B9-5 & & 0,6 & 0,3 & & 0,6 & & & & 0,3 \\
\hline B9-10 & 0,5 & & & & 0,3 & & & & \\
\hline B9-15 & & & 0,2 & & & & & & \\
\hline B9-20 & 0,3 & & & & & & 0,3 & & \\
\hline B9-25 & & & & 0,2 & 0,4 & & 0,2 & 0,4 & \\
\hline B9-30 & & & & 0,5 & & & & & 0,3 \\
\hline
\end{tabular}

Tab. 3 : Détails des taxons hydrophiles.

Tab. 3: Details of hydrophilic taxa. 


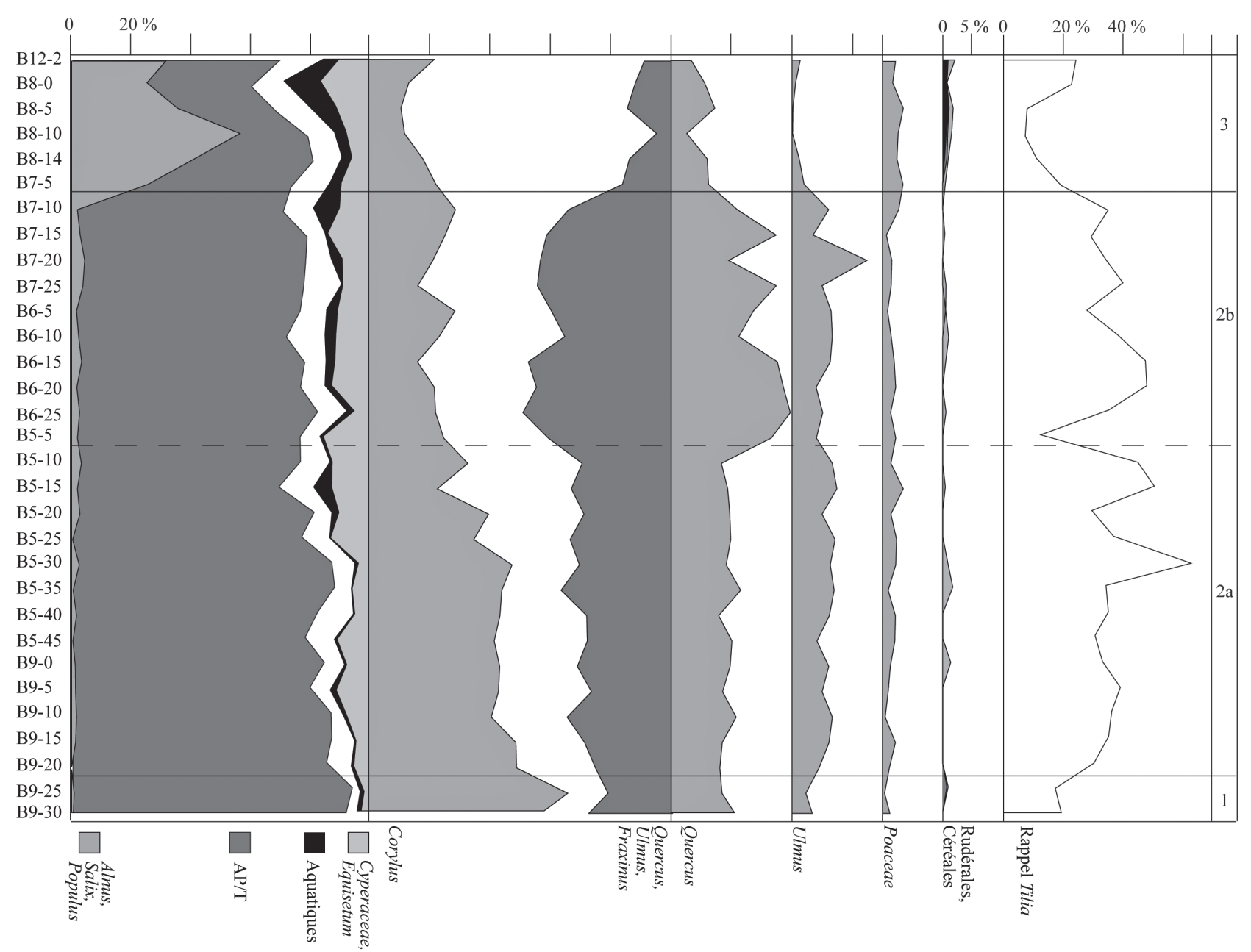

Fig. 6 : Diagramme pollinique partiel (Tilia exclu).

Fig. 6: Partial palynological diagram (excluding Tilia).

Marguerie, 2009, p. 377), ou encore composant d'une roselière (Leroyer et al., 2009). Dans tous les cas, cette famille traduit des conditions locales humides, et ce aussi bien pour des périodes anciennes (Emontspohl \& Vermeersch, 1991) que récentes (Borel et al., 1985). En fond de vallée, le développement d'une prairie humide et la colonisation des lits par une flore hydrophile traduit une plus forte humidité (Leroyer et al., 1997);

- les indices anthropiques, à savoir les céréales ainsi que les adventices et les rudérales (Behre, 1981 ; Brun, 2007 et 2009). Dans ces dernières, sont répertoriés Rumex (oseille), Plantago sp. (plantains), Polygonum $s p$. (renouées), les Urticaceae, les Polygonaceae, les Chenopodiaceae, les Brassicaceae, les Apiaceae et les Caryophyllaceae. Ainsi, chaque phase où ces taxons sont présents a été inventoriée (phases R1 à R8) et discutée de façon qualitative.

\section{4 - RÉSULTATS}

\section{1 - SYNTHÈSE LITHOSTRATIGRAPHIQUE}

La stratigraphie est la suivante, de bas en haut (fig. 3 et 4, tab. 2).
A - Craie fragmentée (couche 1)

La craie apparaît rapidement sur le versant, sous une faible couverture limoneuse. Elle n'a été observée qu'en bordure du bassin avec une plongée rapide vers l'est.

B - Limon homogène gleyifié (couche 2)

La craie est recouverte d'un limon peu épais, homogène, gleyifié.

C - Limon lité sablo-graveleux (couches 3 à 7)

A proximité de l'axe du talweg, des formations fluviatiles litées, hétérogènes, sont observées.

D - Sol noir (couche 8)

Un horizon limoneux plastique, très sombre se développe au sommet de l'unité $\mathrm{C}$ et en épouse la topographie irrégulière.

E - Silex, matrice limoneuse (couche 9)

L'unité (D) est recouverte d'une formation grossière de gélifracts de silex à matrice limoneuse.

F - Sable et limon plastique (couches 10 à 13)

Sable et limon plastique comblent la zone basse entre la plongée de la craie (A) et sa faible couverture limoneuse $(\mathrm{B})$ et les unités $(\mathrm{C})$ à $(\mathrm{E})$ développées dans l'axe du talweg descendant du versant. Les échantillons prélevés dans cette unité pour analyse palynologique se sont révélés stériles.

G - Tourbe (couches 14 à 19) 


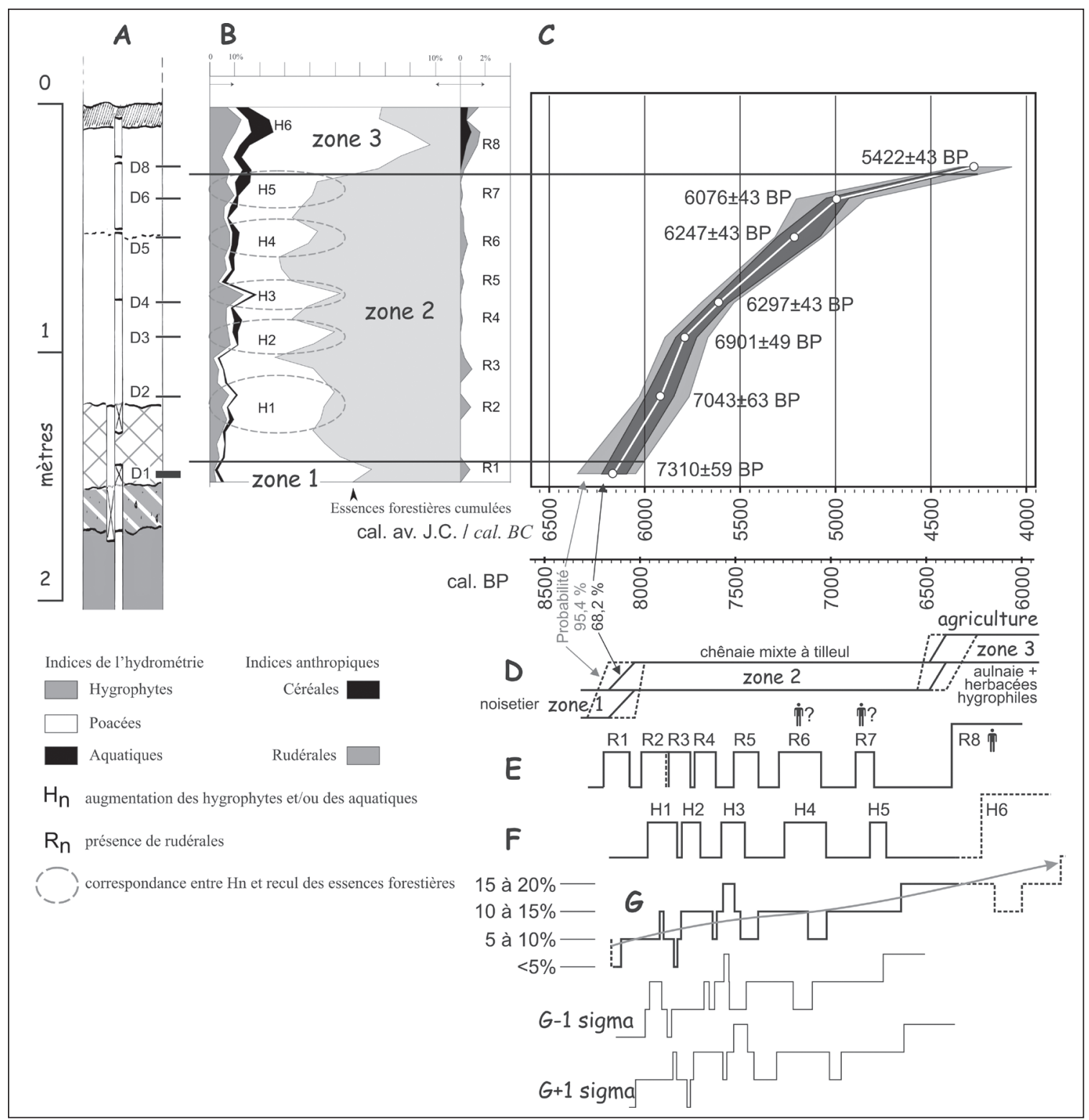

Fig. 7 : Juxtaposition graphique des résultats paléoenvironnementaux.

A/ Profil de la coupe 4 et localisation des datations radiocarbone (sauf D7). B/ Variation des indices polliniques de 1'hydrométrie, de 1'activité anthropique et du couvert forestier (H1 à 6 - augmentation des indices d'humidité, R1 à 8 - présence de plantes rudérales, D1 à 8 - datations radiocarbone, voir tableau 1). C/ Représentation graphique des datations radiocarbone. D/ Succession des palynozones sur une chronologie en années calibrées. E/ Diagramme temporel de présence de rudérales (datation d'après le pic, \pm 1 sigma). F/ Diagramme temporel des correspondances entre indices d'humidité et recul des essences forestières (datation d'après le recul maximum des essences forestières, \pm 1 sigma). G/ Diagramme temporel des variations d'indices d'humidité, par seuil de $5 \%$ (datation d'après la courbe de probabilité maximum, \pm 1 sigma).

Fig. 7: Graphic juxtaposition of palaeoenvironmental results. A/ Profile of record 4 and location of the radiocarbon dates (except D7). B/Variations in palynological indications of wet conditions, human activity and forest cover (H1 to 6 - increase of wet proxies, R1 to 8 - presence of ruderal species, D1 to 8 - radiocarbon dates, see table 1). C/ Graphical representation of the radiocarbon dates. D/ Palynozone succession according to a chronology in calibrated years. E/ Temporal diagram of presence of ruderal species (dates according to peak values \pm 1 sigma). F/ Temporal diagram of correspondence between indications of wet proxies and decline in forest species (dates according to the maximum decline in forest taxa, \pm 1 sigma). $G /$ Temporal diagram of wet condition variations, by $5 \%$ increments (dates according to the maximum probability curve, \pm 1 sigma).

Une tourbe fibreuse, avec quelques bois et noisettes, se développe sur l'unité (F) ; le contact $\mathrm{F} / \mathrm{G}$ présente un très fort pendage vers la vallée. La tourbe est non calcaire mais quelques lits tourbeux très calcaires, avec malacofaune, s'intercalent en limite ouest. Quelques silex taillés ont été observés en partie supérieure de la tourbe. Un tronc de chêne, long de $12,80 \mathrm{~m}$ et d'un diamètre maximal de $0,88 \mathrm{~m}$, était conservé en position horizontale dans les niveaux hauts de la tourbe. L'arbre était positionné perpendiculairement à la zone humide du talweg, occupant une grande partie de sa largeur.
Le dépôt de tourbe bénéficie d'une étude palynologique détaillée (cf. 4.2) et d'un ensemble de datations radiocarbone (fig. 4 et tab. 1) cohérent. Les formations organiques observées (unité G) se développent sur environ 1,5 millénaire (entre environ 6300 et 4000 avant J.-C.). Le bilan sédimentaire est de l'ordre de $11 \mathrm{~cm}$ par siècle depuis la base des niveaux organiques jusqu'au milieu de la couche 16 , elle chute ensuite à environ $4 \mathrm{~cm}$ par siècle puis à moins de $2 \mathrm{~cm}$ par siècle (milieu de la couche 17).

$\mathrm{H}$ - Limon gris (couche 20) 
Un limon gris peu épais scelle le bord de la tourbe. Discrètement litée, l'unité contient du charbon de bois et des éclats de silex taillés.

\section{I - Sol noir (couche 21)}

L'ensemble de la zone est scellé par un horizon limoneux plastique, très sombre, contenant quelques tessons de céramique du haut Moyen Âge et des fragments de tuiles gallo-romaines.

$\mathrm{J}$ - Limon, silex, structures (couches 22 à 28)

Le talweg est comblé par plusieurs mètres de limon, avec des passages de cailloutis de silex, et des remblais récents. La base de l'unité montre plusieurs générations de limon poudreux laminé et remanié en blocs (labours de colluvions limoneuses/croûtes de battance).

\section{2 - SÉQUENCE POLLINIQUE}

Suivant les couches rencontrées, la richesse sporopollinique est tout à fait variable.

Seules les couches organiques $(14,16,17$ et 21 , soit unités G et I), sont riches en pollen (fig. 8). En moyenne, 419 grains de pollen ont été comptés par échantillon. En revanche la diversité taxonomique n'est pas très élevée même si elle est statistiquement suffisante (21 taxons en moyenne). La végétation enregistrée semble assez peu diversifiée. L'hypothèse d'un problème de conservation peut être rejetée car les valeurs des concentrations absolues sont élevées (27939 en moyenne) ; de plus, les taxons habituellement surreprésentés en cas de conservation différentielle (Havinga, 1984) sont, au contraire, ici fort discrets. Ces échantillons peuvent donc être considérés comme fiables.

Seule la partie supérieure de la séquence stratigraphique enregistre donc l'évolution de la végétation. Elle correspond aux dépôts les plus organiques. Par ailleurs, les valeurs des concentrations absolues totales présentent une très grande variabilité d'un échantillon à l'autre. Cela limitera de fait l'interprétation du comportement de chacun des taxons, dont les variations ne sont significatives qu'à concentration totale constante. Cette séquence (fig. 5), qui comporte des caractéristiques assez homogènes sur l'ensemble, se divise en trois zones, du fait de variations dans les assemblages polliniques.

La strate arborée est particulièrement bien représentée dans ce diagramme (le rapport AP/NAP atteint en moyenne $85 \%$ ). Au sein de celle-ci, les essences forestières telles que Tilia, Quercus, Ulmus, et quelques Fraxinus, occupent une place importante (leurs taux cumulés sont à $52 \%$ en moyenne, mais peuvent atteindre près de $75 \%$ ). Vient ensuite Corylus (noisetier), avec une moyenne de 21,3\%. D'autres taxons héliophiles sont également présents, mais avec des taux qui ne dépassent que rarement les $1 \%$. Pinus (pin) présente une courbe régulière, autour de 3,6\%.

Parmi les ligneux hygro- à mésohygrophiles - groupements de l'aulnaie des vallées marécageuses, et celui des forêts riveraines sur alluvions (Bournérias, 1968), Salix (saule) et Populus (peuplier) restent discrets tout au long du diagramme, tandis que la courbe de Alnus (aulne), continue mais faible sur une grande part de la séquence, va très nettement se développer dans la partie supérieure.

La strate herbacée est dominée par les Cyperaceae ( $6 \%$ en moyenne), les Poaceae (2,5\% en moyenne) et les aquatiques $(2,2 \%)$.

Les indices polliniques de présence de l'homme sont très ténus : les céréales ne seront attestées que dans la partie supérieure, tandis que les rudérales sont enregistrées sur l'ensemble de la séquence, mais de manière très sporadique et sans jamais dépasser $1 \%$.

\subsection{1 - Zone 1 (échantillons B9-30 et 25)}

La zone 1 se développe sur les deux seuls niveaux inférieurs, soit la base de la couche 14. Le rapport AP/ NAP, de 93,3\% en moyenne, est très élevé. Corylus atteint ici un taux maximum (49,3\%); il est secondé de quelques autres ligneux héliophiles tels Betula (bouleau) et Hedera (lierre), dont les taux sont inférieurs à $1 \%$. Ce dernier se développe certes en milieu ombragé, mais il ne fleurit que s'il est exposé à la lumière ; la présence de son pollen le classe alors parmi les héliophiles. La ripisylve est très faiblement perçue (Alnus et Populus ont des taux inférieurs à $1 \%$ ), tandis que les essences forestières totalisent déjà 39,3\%. Elles présentent des taux relativement équilibrés entre Quercus et Tilia (respectivement 15,9 et $18,5 \%$ ), Ulmus étant plus discret avec 4,5\%.

La strate herbacée, très peu diversifiée, est dominée par les Poaceae et les Cyperaceae (1,4 et 1,8\%). Des aquatiques sont présentes de manière sporadique. La présence de rudérales (Rumex et Brassicaceae) est notée au niveau B9-25.

\subsection{2 - Zone 2 (échantillons B9-20 à B7-10)}

La seconde zone se développe sur la majeure partie du diagramme. Le rapport AP/NAP atteint maintenant de fortes valeurs, avec $86,5 \%$ de moyenne. Corylus a nettement reculé ; il n'atteint plus que 20,9\%. En revanche, les essences forestières se sont développées et totalisent un taux record de 59,7 \%, où Tilia est maintenant l'essence nettement prédominante $(37,1 \%)$ devant Quercus $(15,1 \%)$ et Ulmus $(7,5 \%)$.

La courbe de Tilia n'est pas régulière. Elle présente en effet une succession de reculs et d'accroissements. A chacun de ces minima correspond une augmentation des taux de Quercus. Certains sont vérifiés lorsque Tilia est sorti de la somme de base (fig. 6) et plus rarement en concentrations absolues (fig. 8). Par ailleurs, la courbe du cumul de l'ensemble des essences forestières présente des fluctuations qui s'avèrent le plus souvent inversement proportionnelles à celles de la courbe de Corylus. Ces variations se retrouvent également lorsque la somme de base est restreinte, les fluctuations des courbes s'imbriquant de façon quasi parfaite (fig. 6).

De plus, sur la base essentiellement des courbes de Quercus et Corylus, il est possible de proposer une sous- 


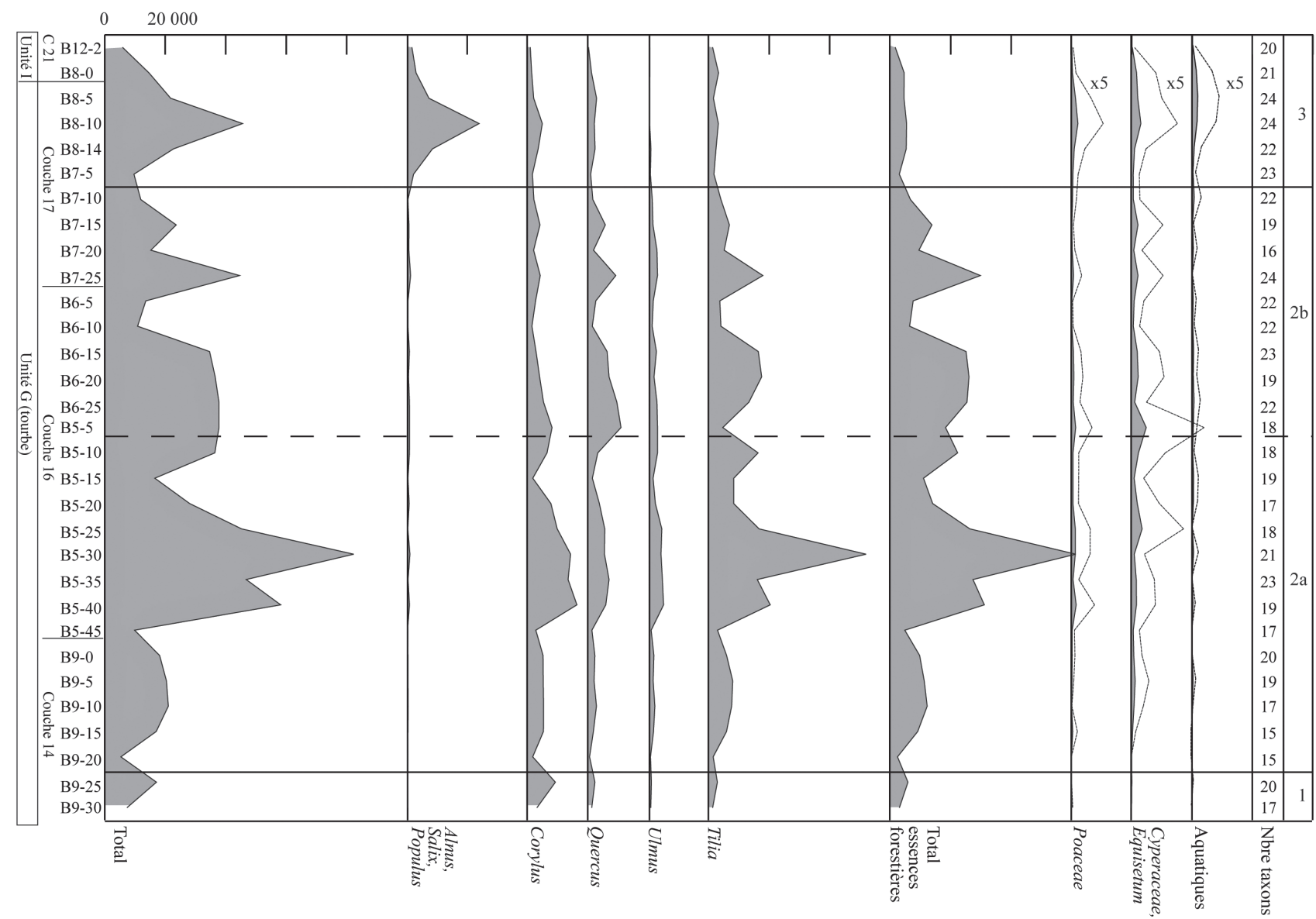

Fig. 8 : Diagramme de concentrations absolues et diversité. Fig. 8: Diagram of absolute concentrations and diversity.

zonation. Ainsi, dans un premier temps, zone $2 \mathrm{a}$, Corylus atteint encore $34,3 \%$ de moyenne, contre $15,1 \%$ en $2 b$, tandis que Quercus ne dépasse pas $15,1 \%$ en 2 a mais pourra atteindre $29,1 \%$ en $2 b$.

Sur l'ensemble, les taux des Poaceae restent stables ( $2 \%$ en moyenne). Les hygrophiles (Cyperaceae et Equisetum - prêles -) se sont développées (5,9\% en moyenne, avec un maximum à $13 \%$ ). Les aquatiques vont également montrer une progression, faible mais régulière (jusqu'à atteindre $6 \%$ ). Quant aux rudérales, elles sont enregistrées de manière assez régulière, voire cyclique, tout en restant avec des valeurs inférieures à $1 \%$. Pour la plupart d'entre elles, il s'agit de familles à larges spectres écologiques, dont certaines espèces seulement sont des rudérales (Brun, 2007, 2009). C'est pourquoi, chacune de ces occurrences se doit d'être discutée.

\subsection{3 - Zone 3 (échantillons B7-5 à B12-2)}

Les assemblages polliniques évoluent. Le rapport AP/ NAP a légèrement régressé (75,8 \% en moyenne). Un rapide recul des taux de l'ensemble des essences forestières profitera, dans un premier temps, aux héliophiles (Betula et Corylus) qui présentent une légère augmentation. Mais, très rapidement, Alnus prend le relais et devient l'essence dominante (jusqu'à 51,6\%).
En ce qui concerne la strate herbacée, les courbes de Poaceae, Cyperaceae et des aquatiques progressent ; leurs taux respectifs sont de 4,5\%,8,2\% et 5,6\% de moyenne. Les rudérales ne présentent pas d'augmentation $(0,6 \%$ de moyenne), mais les céréales seront enregistrées de façon continue, avec des taux qui restent cependant très discrets (inférieurs à $1 \%$ ).

En fin de zone (couche 21), Alnus s'efface devant un nouvel essor de Tilia, phase où les hygrophytes et les aquatiques présentent leurs taux les plus élevés.

\section{5 - INTERPRÉTATION}

\section{1 - HYPOTHÈSES D’ÉVOLUTION DE LA ZONE}

La stratigraphie et les éléments de datation permettent de proposer le scénario d'évolution suivant (fig. 9 et 10).

\subsection{1 - Phase 1 - Pléniglaciaire : cône de déjection au pied du versant (unité C)}

La zone observée se situe sur la bordure d'un cône de déjection provenant du versant. Le cône débouche dans le fond de vallée pléniglaciaire, avec éventuellement des phénomènes d'inter-stratification entre les deux ensembles morpho-sédimentaires. 


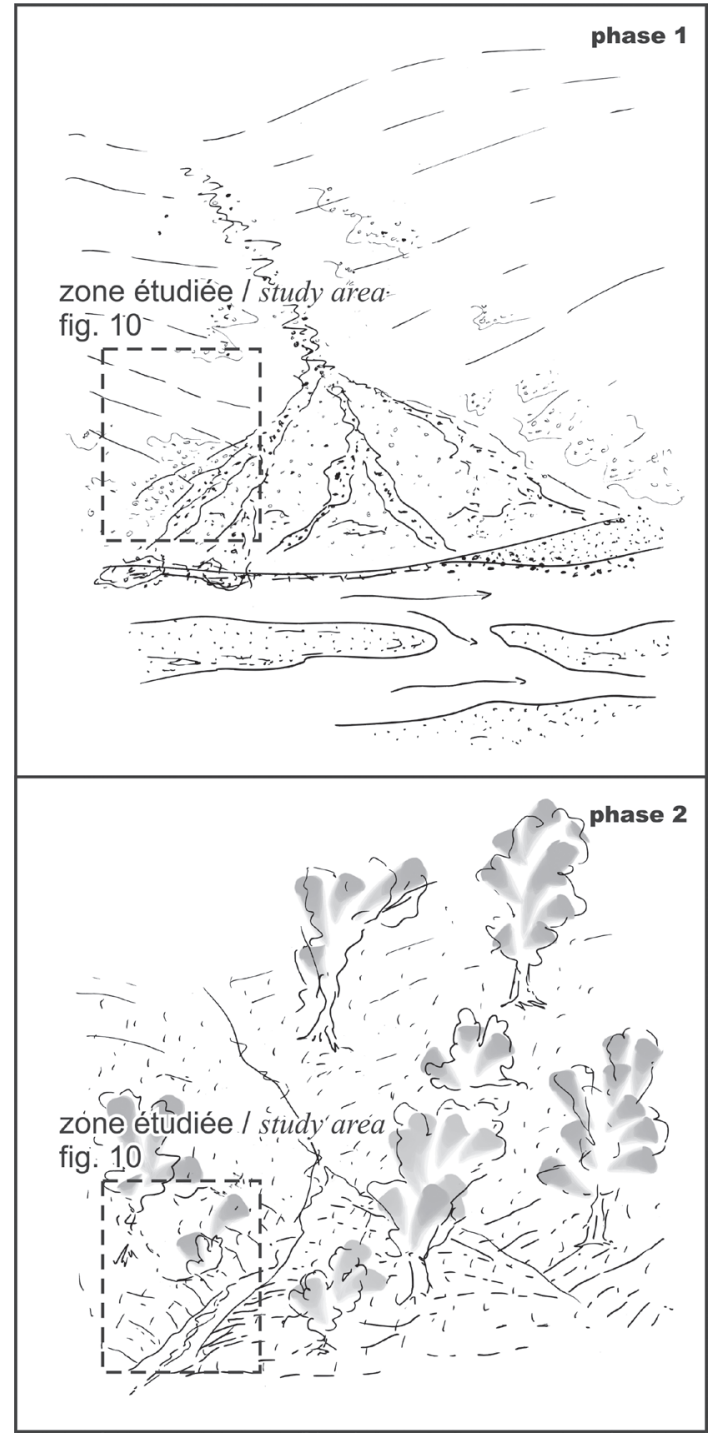

Fig. 9 : Hypothèse d'évolution géodynamique de la zone d'étude, phases 1 et 2.

Phase 1 : dépôt de l'unité C, cône de déjection/fond de vallée, Pléniglaciaire. Phase 2 : formation de l'unité $\mathrm{D}$, sol interstadiaire tardiglaciaire. Fig. 9: Hypothesis of geodynamic evolution of the studied area, phases 1 and 2 .

Phase 1: deposition of unit $C$, alluvial fan/valley bottom, Pleniglacial. Phase 2: formation of unit D, Lateglacial interstadial soil.

\subsection{2 - Phase 2 - Interstade tardiglaciaire : dévelop- pement d'un sol (unité D)}

Un petit sol, présent seulement au nord, épouse la topographie du talweg. Sa position, au-dessus des dépôts grossiers attribués au Pléniglaciaire et son faciès (horizon plastique sombre) permettent de proposer une corrélation avec un sol interstadiaire du Tardiglaciaire observé dans la même position dans la vallée et daté par la malacologie à Bruay-sur-Escaut (Locht, 2005). Une corrélation avec le sol Allerød fréquemment observée dans les fonds de vallée du Bassin parisien peut être proposée (Pastre et al., 2002). Le sol est observé au nord tandis qu'en rive sud, la craie fragmentée du versant affleure immédiatement. La topographie qui a permis l'enregistrement des dépôts postérieurs semble donc être déjà constituée.

\subsection{3 - Phase 3 - Dryas récent : déstabilisation du versant (unité E)}

Le petit sol attribué à un interstade du Tardiglaciaire est recouvert par des dépôts grossiers. Les apports grossiers sont localisés exclusivement dans la partie nord de l'observation et donc dans l'axe du talweg sensible sur le versant. Il s'agit vraisemblablement de dépôts liés à une phase d'activité fluviatile sur le versant. Ceci pourrait concorder avec l'importante déstabilisation des versants au cours de la péjoration froide du Dryas récent observé fréquemment dans un contexte de plateau crayeux (Pastre et al., 2002).

5.1.4 - Phase 4 - Première partie de l'Holocène : accumulation colluviale (unité F)

Une accumulation limono-argileuse grise constitue le bilan de la première partie de l'Holocène. L'absence de pollen conservé (pourtant dans un sédiment limoneux sous la nappe, a priori propice à leur conservation) et la très forte pente observée au sommet de l'unité confortent l'interprétation d'une mise en place colluviale.

5.1.5 - Phase 5 - Atlantique ancien : remontée rapide de la nappe et ennoiement par la tourbe (partie inférieure de l'unité $G=$ couches 14 à 16,18 et 19)

Le limon fin gris est surmonté par une tourbe fibreuse avec bois. La présence de tourbe implique le développement d'un milieu anaérobie (remontée de la nappe). Le fond de vallée atteint donc la zone observée et la sédimentation organique remonte dans le petit talweg de manière relativement rapide. Le fait concorde avec le fort développement de tourbières atlantiques dans le fond de la vallée de l'Escaut. Quelques éléments d'industrie lithique épars témoignent de passages humains. Toutefois, aucun vestige structuré n'a été observé.

Un écoulement provenant du versant continue à s'exprimer par des lits limoneux calcaires inter-stratifiés avec les tourbes. Il s'agit de la couche 19 (tourbe et lits limoneux) qui forme une variation latérale du faciès tourbeux. Il s'agit probablement d'écoulements sporadiques dans le talweg lors d'épisodes pluvieux. Le faciès, peu développé en épaisseur, semble couvrir toute la période de formation de la tourbe et est limité à la jonction avec le versant.

5.1.6 - Phase 6 - Atlantique récent : remontée lente de la nappe et premiers indices d'agriculture (partie supérieure de l'unité G)

Dans les derniers décimètres de la tourbe, le taux de sédimentation devient beaucoup plus faible. C'est dans ce contexte qu'un chêne multicentenaire tombe dans la tourbière après 4930 avant J.C. La partie sommitale de la tourbe enregistre les premiers indices d'agriculture, à partir d'environ $5422 \pm 43$ BP (soit 5 031-4 800 ans av. J.-C.).

Il n'y a pas de terminus post-quem pour la fin du développement de la tourbière si ce n'est un dépôt colluvial 

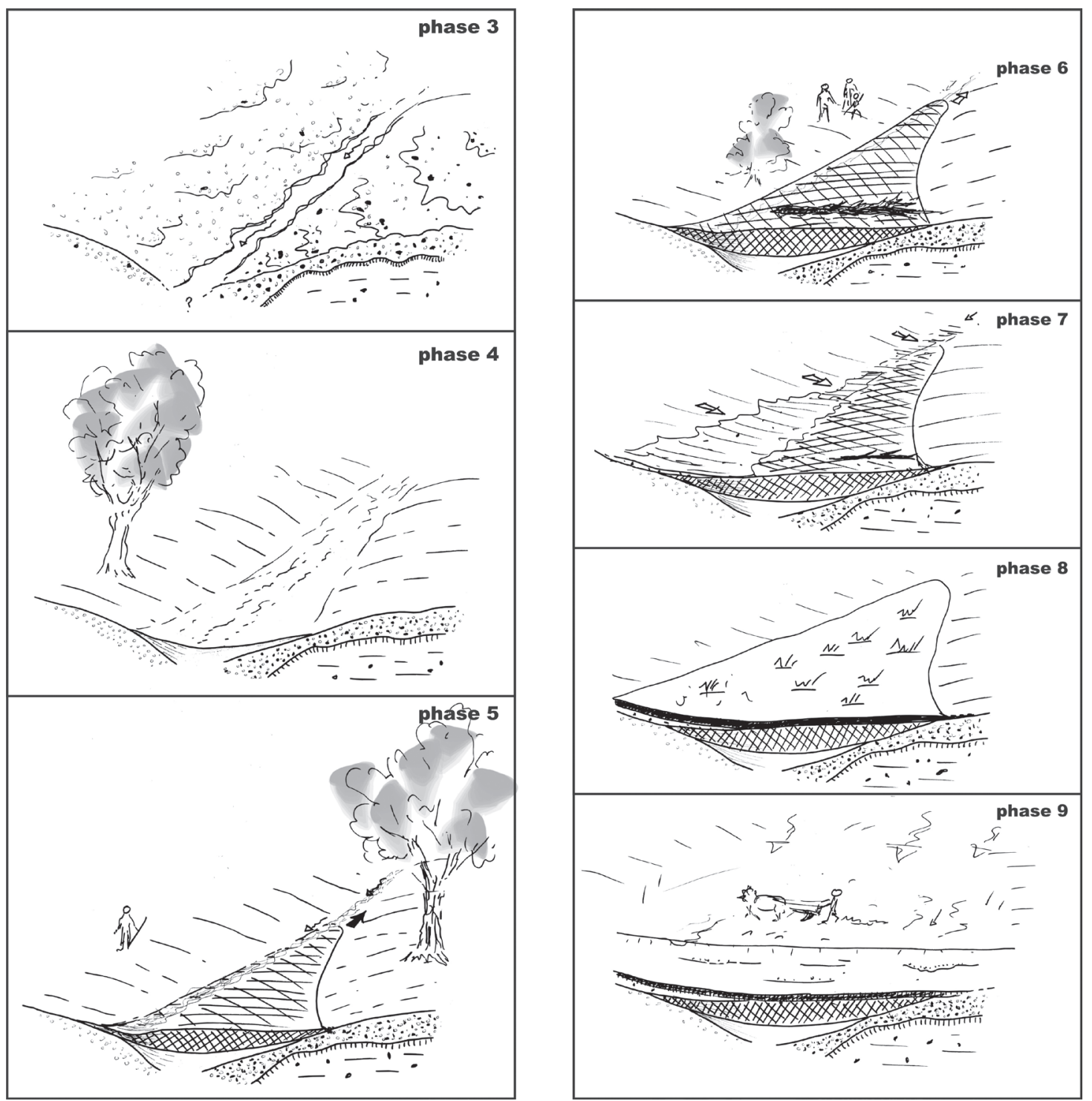

Fig. 10 : Hypothèse d'évolution géodynamique de la zone d'étude, phases 3 à 9.

Phase 3 : dépôt de l'unité E, érosion, apport de silex depuis le versant, Dryas récent. Phase 4 : dépôt de l'unité F, colluvions en provenance du versant, première partie de l'Holocène Phase 5 : développement de l'unité $G$, remontée de la nappe et envahissement du talweg par une tourbière provenant du fond de vallée, écoulements sporadiques depuis le versant, Atlantique. Phase 6 : fin du dépôt de l’unité G, ralentissement du développement de la tourbe. Phase 7 : dépôt de l'unité H, colluvions. Phase 8 : dépôt de l'unité I, formation d'un sol de marais, tessons du Haut Moyen Âge. Phase 9 : dépôt de l'unité J, mise en culture et colluvions.

Fig. 10: Hypothesis of geodynamic evolution of the studied area, phases 3 to 9. Phase 3: deposition of unit E, erosion, flint input from the slope, Younger Dryas. Phase 4: deposition of unit F, colluvia from the slope, early Holocene. Phase 5: development of unit $G$, water table rise and talweg flooding by peat coming from the valley bottom, sporadic flows from the slope, Atlantic. Phase 6: end of the deposition of unit G, slowdown of peat development. Phase 7: deposition of unit H, colluvia. Phase 8: deposition of unit I, formation of a marsh soil, Early Middle Age potsherds. Phase 9: deposition of unit $J$, cultivation and colluvia.

en rive droite (phase 7) et la présence de matériel céramique du haut Moyen Âge associé au sol qui scelle l'ensemble (phase 8 ). Toutefois, il est probable que, comme ailleurs dans la vallée (Ruchard et al., 1992), la tourbification massive n'ait plus lieu au cours du SubboréalSubatlantique.

\subsection{7 - Phase 7 - Colluvions avec matériel archéolo- gique (unité $\mathbf{H}$ )}

En rive droite, une unité peu épaisse, limoneuse, scelle la tourbe. Elle a livré des éléments d'industrie lithique, un peu de faune, quelques tessons (Néolithique moyen?) et des charbons de bois. En l'absence de calage chronologique précis, il est délicat de savoir si le dépôt est rapide ou lent. Dans les deux cas, il est interprété comme un apport colluvial depuis le versant. Le fait concorde avec le développement de l'agriculture et la dénudation du sol.

\subsection{8 - Phase 8 - Petit sol de marais et stabilité morphologique (unité I)}

Un horizon de sol argileux et noir, scelle la tourbière. La sédimentation est fine, argileuse, mais avec un bilan sédimentaire pratiquement nul pendant une longue période comme l'indique la présence de matériel céramique du 
haut Moyen Âge. Le niveau est interprété comme un sol développé en contexte humide mais sans apport notable de sédiment depuis le cours d'eau ou le versant. Il s'agit vraisemblablement d'un sol de marais développé dans un contexte de stabilité morphologique.

\subsection{9 - Phase 9 - Fortes colluvions historiques et labours (unité J)}

Postérieurement au dépôt renfermant du matériel du haut Moyen Âge, une épaisse couche de colluvions limoneuses, avec passées de cailloutis, scelle le talweg. Elle résulte de la mise en culture du versant et recèle des indices de labour. Cet apport massif, de l'ordre de trois mètres, ennoie le talweg et tend à l'estomper. Deux fondations de bâtiments et une petite terrasse artificielle, maintenue par un muret de soutènement, témoignent enfin des derniers aménagements de la zone après la fermeture du talweg. Ces derniers, datables de la fin $\mathrm{Xv}^{\mathrm{e}}-\mathrm{XVI}^{\mathrm{e}}$ siècles, pourraient être associés à la culture de la vigne, à l'origine de la toponymie du site.

\section{2 - INTERPRÉTATION DES PALYNOZONES}

L'ensemble de la séquence ayant fourni des résultats, soit les unités $G$ et I, présente une certaine homogénéité (fig. 5). Les variations observées tout au long de la séquence peuvent être considérées comme bien réelles et non pas simples effets de statistiques, puisqu'elles sont également enregistrées en concentrations absolues (fig. 8).

Sur la totalité de la séquence, l'environnement apparaît fortement boisé, et constitué principalement d'une chênaie mixte à tilleul dominant, au sein de laquelle Ulmus et quelques Fraxinus trouvent également leur place.

Des phases de recul de cet espace forestier sont observées et sont surtout imputables à des diminutions de la courbe de Tilia. Ces ouvertures ponctuelles vont profiter aux héliophiles (ici surtout Corylus), qui régressent ensuite devant une nouvelle progression de la forêt. Ce phénomène apparaît de manière assez cyclique tout au long de la séquence.

A ces phases de recul, correspond parfois un enregistrement de rudérales qui pourraient témoigner d'une présence humaine ; toutefois, aucun de ces niveaux n'a révélé la présence de micro-charbons de bois qui auraient pu suggérer des incendies (qu'ils soient d'origine humaine ou non). La distinction entre, d'une part, les taxons qui sont réellement des rudérales, et, d'autre part, ceux dont certaines espèces seulement témoignent d'une présence humaine, devient alors indispensable. Aussi, l'absence de présence humaine clairement attestée incite à rechercher d'autres indices de phénomènes qui pourraient avoir une incidence sur le retrait de la chênaie mixte, et en particulier sur la courbe de Tilia. Il se trouve que ces phases de retrait correspondent ici à un accroissement des indices d'humidité, même si les proportions sont encore faibles (H1 à H5, fig. 7). Les changements des conditions édaphiques impliquent des variations de contraintes sur le développement de l'espace forestier. En effet, si Fraxinus, Quercus et Ulmus peuvent vivre en plaine alluviale, sur des sols humides (Emontsphol, 1994), et que l'on retrouve même ces deniers associés aux forêts riveraines sur alluvions (Bournérias, 1968), Tilia, lui, ne tolère au mieux qu'un sol frais (Rameau et $a l ., 1989)$. Les variations des conditions édaphiques ont donc une incidence directe sur sa courbe.

Par ailleurs, cette essence est prépondérante sur la séquence, alors que, durant l'Atlantique ancien, elle ne dépasse pas quelques pourcentages en Île-de-France (Leroyer, 1997) et dans la plupart des diagrammes de l'Aisne (Boulen, 1995, 1997, 2001, 2011a), elle atteint 20 à $30 \%$ dans le Nord (Deschodt et al., 2004, 2006), et présente des taux aussi importants qu'au Vignoble dans certains diagrammes en Belgique (Munaut, 1967). Cet auteur souligne l'importance prise par Tilia dans la composition des forêts atlantiques sur sable - ce qui expliquerait son importance dès l'Atlantique ancien, dans les zones polliniques antérieures à environ 7500 ans cal. BP, à Soupir/Moussy-Verneuil où la plaine alluviale s'inscrit dans des sables sparnaciens (Boulen, 2011b) - ainsi que sur sols calcaires (comme c'est le cas au Vignoble).

Enfin, les datations radiocarbone (tab. 1) placent l'ensemble de la séquence, soit 1,5 m de sédimentation, durant l'Atlantique, ce qui représente un enregistrement bien détaillé de cette chronozone. Pour la région, une telle épaisseur est exceptionnelle. En effet, quand elle est enregistrée, cette chronozone se limite souvent à 20-25 cm de sédimentation ; c'est le cas sur la Deûle, à Houplin-Ancoisne (Boulen in Praud et al., sous presse) et dans différentes séquences de l'Aisne (Boulen, 1995, 2001, 2011a). Elle atteint plus exceptionnellement 50 à $60 \mathrm{~cm}$, comme à Lille (Deschodt et al., 2006) et dans d'autres séquences de l'Aisne (Boulen, 1997, 2011b).

\subsection{1 - Zone 1 (échantillons B9-30 et 25)}

La datation radiocarbone à $7310 \pm 59$ BP (environ 8100 ans cal. BP, tab. 1), soit durant l'Atlantique ancien est en adéquation avec les résultats polliniques : les essences forestières caractéristiques d'une chênaie/tillaie sont déjà bien implantées. Par ailleurs, Corylus présente ici un taux encore très élevé, réminiscence de la végétation prédominante durant le Boréal. Mais il est encore souvent important sur une grande part de l'Atlantique (Boulen, 2011b).

La strate herbacée est peu représentée, et peu diversifiée. Elle présente les caractéristiques d'une prairie humide à Poaceae et Cyperaceae. Un premier enregistrement de rudérales est déjà présent (phase R1 dans la figure 7). Celle-ci paraît en corrélation avec un recul des essences forestières.

\subsection{2 - Zone 2 (échantillons B9-20 à B7-10)}

Les cinq dates radiocarbone (tab. 1) obtenues sur la zone témoignent d'une sédimentation relativement régulière (fig. 7), toujours durant l'Atlantique ancien. 
La chênaie/tillaie, largement dominée par Tilia durant toute la zone 2 malgré des fluctuations cycliques de sa courbe, va atteindre ici un optimum qui n'est cependant pas constant (voir l'alternance des pics de Quercus et Tilia et phases de recul de l'ensemble au profit de Corylus). Si, dans un premier temps (zone 2a), Corylus tient encore une place importante, il s'efface ensuite devant le développement de Quercus (zone 2b) et la fermeture du milieu. Parallèlement, l'aulnaie se développe légèrement en $2 \mathrm{~b}$. Si des indices anthropiques sont enregistrés, ils restent cependant bien ténus. En revanche, à la plupart de ces reculs de la chênaie correspond une petite augmentation des hygrophytes ( $\mathrm{H} 1$ à $\mathrm{H} 5$ dans la figure 7).

\subsection{3 - Zone 3 (échantillons B7-5 à B12-2)}

Une aulnaie va occuper le fond de vallée. Elle se développe très rapidement, mais de façon continue sur une quinzaine de centimètres, ce qui tendrait à exclure l'hypothèse d'un hiatus sédimentaire.

En même temps, un développement des rudérales conjointement à l'apparition de la courbe des céréales, traduisent une activité agricole qui a pour conséquence un très net recul des essences forestières, que l'on constate également dans les courbes construites sans Alnus.

En fin de zone, sur la couche 21, l'aulnaie, qui a malgré tout besoin d'un sol bien drainé, décroît et laisse place à une végétation herbacée de milieu humide. Pendant ce temps, le tilleul montre un nouvel essor.

Le développement généralisé des aulnaies est récurrent à l'Atlantique récent, où les premières traces d'agriculture néolithique avec l'enregistrement des céréales sont nettement perçues ici.

\section{6 - DISCUSSIONS}

La séquence palynologique permet de mettre en évidence des variations du couvert forestier, de l'hydrométrie et de l'activité humaine (fig. 7) d'environ 7300 à 6000 BP.

\section{1 - LA PRÉSENCE DE RUDÉRALES ET LE LIEN AVEC L'ACTIVITÉ HUMAINE?}

Comme cela a été souligné, des grains de pollen de plantes rudérales sont enregistrés, certes de manière

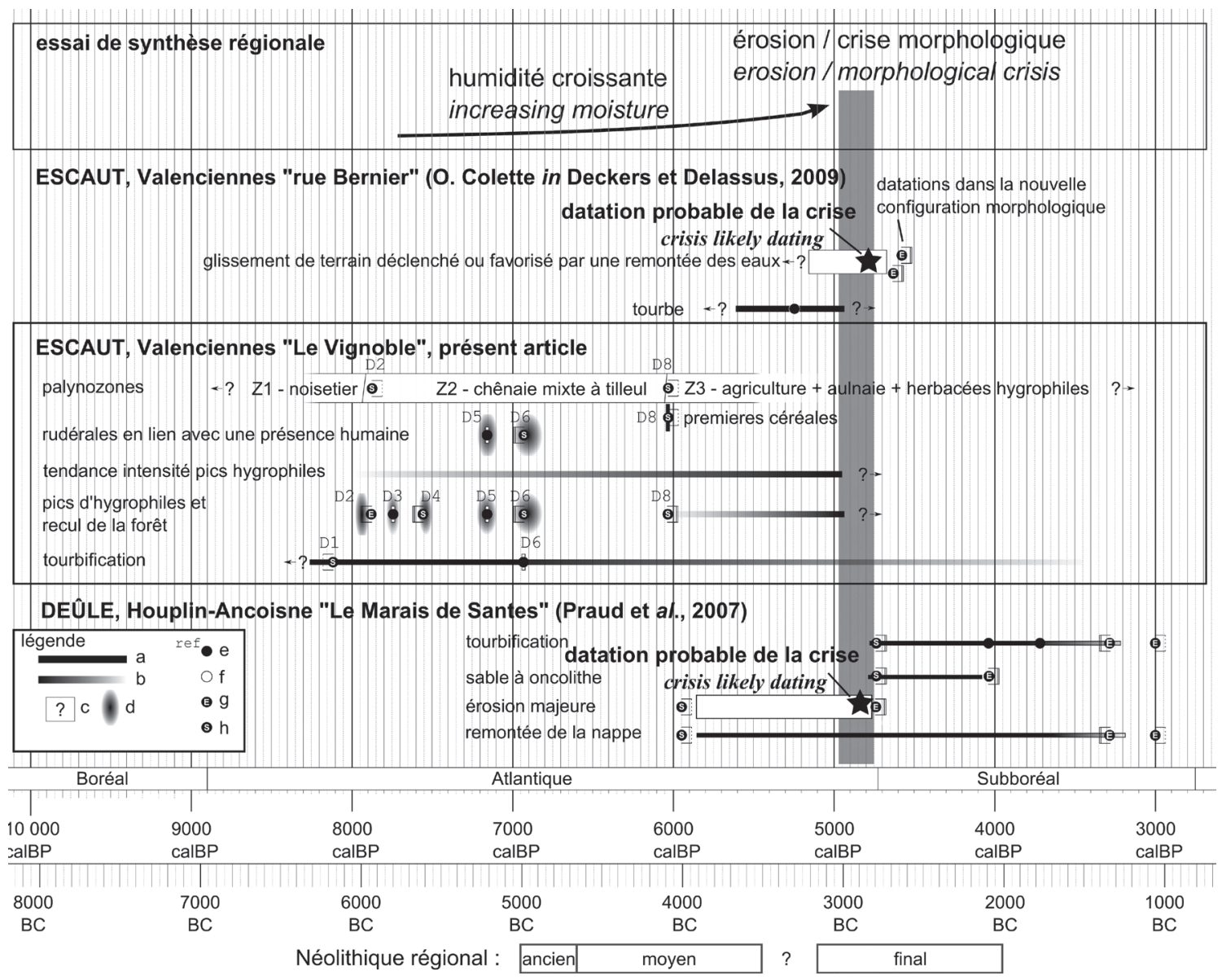

Fig. 11 : Frise chronologique et corrélation d'événements régionaux.

a/ Fourchette chronologique d'un événement. b/ Fourchette chronologique d'un événement d'intensité variable. c/ Evénement court mais terminus mal connus. d/ Evénement de durée intermédiaire (décennies ?). e/ Datation radiocarbone (et référence). f/ Autre datation (archéologie, etc.). g/ Datation de la fin de l'événement. h/ Datation du début de l'événement.

Fig. 11: Timeline and correlation of regional events. a/ Chronological range of an event. b/ Chronological range of a variably intense event. c/ Short term event with a poorly defined terminus. d/ Intermediate-term event (decades?). e/ Radiocarbon date (and reference). f/ Other date (archaeology, etc.). $g /$ Date of the event terminus. $h$ / Inception date of the event. 
ténue, mais régulière, voire cyclique tout au long de la séquence (fig. 7). Ces huit phases observées sont à discuter en fonction de la «qualité » de ces indices : s'agit-il réellement de rudérales ou de familles dont certaines de leurs espèces témoignent effectivement d'une présence humaine ? Cette présence a-t-elle un impact sur l'environnement boisé ? Et les variations de ce dernier peuvent-elles être imputées à un autre phénomène que l'activité humaine?

Dans la première occurrence (échantillon B9-25, phase R1), il s'agit de Rumex (oseille) qui peut être considérée comme une plante rudérale (Brun, 2007). Elle serait alors ici le témoignage d'une présence humaine. Cependant, la baisse des pourcentages des essences forestières ne peut pas encore être interprétée ici comme la conséquence directe de défrichements. En effet, ce recul ne correspond vraisemblablement pas à une réalité, mais à une dérive statistique, car il n'apparaît pas en concentrations absolues (fig. 8). Il ne semble imputable qu'au seul pic de Corylus qui réduit la représentation de l'ensemble des autres taxons, dont les essences forestières.

Pour les occurrences R2 à R5, non seulement les rudérales enregistrées sont des familles à large écologie, mais de plus, les reculs de la représentation des arbres enregistrés sur cette partie correspondent à une légère augmentation des indicateurs d'humidité. Ainsi, les courbes des hygrophytes et aquatiques, d'une part, et des essences forestières, d'autre part, présentent une correspondance quasi parfaite, les unes reculant lorsque les autres se développent, et inversement (fig. 6 et 7).

Dans les spectres polliniques qui correspondent aux phases R6 et R7, il est possible d'envisager une présence humaine de part l'enregistrement de pollen de rudérales (Urticaceae et autres rudérales à écologies plus larges, puis Rumex). En revanche, une nouvelle fois, le recul de la chênaie/tillaie peut aussi être en relation avec le développement d'un bas-marais, et non avec une activité humaine.

Enfin, l'ensemble des échantillons qui composent la zone pollinique 3 témoigne de la présence de céréales et de rudérales (phase R8) ; l'activité humaine est ici incontestable. Par ailleurs, la forte chute de la représentation de la chênaie reste nette en excluant Alnus de la somme de base, ainsi qu'en concentration absolue (fig. 8). De plus, le minimum de la chênaie correspond à une phase où les indices d'humidité sont plutôt en retrait (entre les pics H5 et H6). Cela autorise à considérer ce recul comme l'enregistrement d'un défrichement, lié à l'activité agricole. La base de la zone est datée à $5422 \pm 43 \mathrm{BP}$, soit environ 6300 ans cal. BP, attribuant ces occupations au Néolithique moyen.

En considérant les taxons répertoriés de manière qualitative, et en cherchant d'autres indices dans la végétation enregistrée pour expliquer ces phases à rudérales, il a été possible de relativiser chacune d'entre elles et, pour la plupart, rejeter l'hypothèse d'une présence humaine. Il existe en revanche une bonne corrélation entre les reculs de la tillaie et les augmentations des indices d'humidité, cette essence supportant mal les sols trop humides (Rameau et al., 1989).

\section{2 - DES CONDITIONS DE PLUS EN PLUS HUMIDES AU COURS DE L'ATLANTIQUE?}

Les $6^{e}$ et $5^{\mathrm{e}}$ millénaires (entre 8000 et 6000 ans cal. BP) voient le retour périodique de conditions plus humides certes peu marquées mais néanmoins présentes $(\mathrm{H} 1$ à H6, fig. 7). L'enregistrement a lieu dans la zone humide, hors chenal et à l'abri de l'activité directe du cours d'eau (ce qui n'exclut pas, de manière anecdotique, quelques écoulements intermittents depuis le versant, marqués par des lits limoneux en bordure de la tourbière). Ainsi, le postulat est posé qu'il correspond bien à l'image de la végétation et non à l'influence d'une évolution paléogéographique locale qui aurait été marquée par des hiatus ou des variations de la sédimentation (travertin ou remaniement de travertin, abondant dans cette section de vallée).

Les indices de conditions plus humides sont enregistrés tandis que la croissance de la tourbière décline fortement et sans que soient observées des traces d'érosions ou d'apports détritiques importants. Ils sont les plus élevés dans la palynozone 3 , avec les indices d'agriculture, l'ouverture de la forêt, le développement d'une aulnaie et même, à la fin de l'enregistrement, la dégradation de cette aulnaie au profit d'une zone humide plus ouverte.

\section{3 - DYNAMIQUE MORPHO-SÉDIMENTAIRE À L'ATLANTIQUE}

L'évolution morpho-sédimentaire de "l'Étang du Vignoble » est similaire à celle enregistrée dans la Somme où, depuis le Boréal, le fond de vallée est occupé par une large tourbière avec chenaux résiduels latéraux (Antoine et al., 2000). Au cours de l'Atlantique, la tourbière est incisée par un nouveau système chenalisé. Dans les deux cas, une déforestation anthropique due à la néolithisation de la région est considérée comme cause possible de cette modification majeure intra-holocène.

\subsection{1 - Le bassin amont de l'Escaut : augmentation de l'humidité et crises érosives ?}

Le ralentissement, à la fin de l'Atlantique, puis l'arrêt du développement tourbeux est conforme à d'autres données proches de la vallée (Ruchard et al., 1992 ; Deschodt, 2002) ; au dépôt extensif de tourbe et de travertin succèdent des formations détritiques en chenaux ou dans la plaine d'inondation. Les limons d'inondation sont absents du site du Vignoble mais largement représentés par ailleurs (Deschodt, 2001). Légèrement en amont du Vignoble, à Rouvignies-Haulchin, la vallée de l'Escaut est envahie par la tourbe. La tourbière est réincisée par un chenal méandriforme (Leriche, 2009). Le sommet de la tourbe fournit un terminus ante-quem à $6962 \pm 72$ BP (environ 7800 ans cal. BP) tandis qu'un élément archéologique dans le chenal fournit un terminus post-quem à $4064 \pm 49 \mathrm{BP}$ (environ 4600 ans cal. BP). En aval de Tournai, dans la partie belge du bassin, de nouveaux chenaux sont incisés à partir d'environ 5000 BP (env. 5700 ans cal. BP) (Kiden, 1991) et une reprise de l'activité fluviatile succède à des écoulements 
extrêmement restreints dans la basse vallée de l'Escaut (Verbruggen \& Kiden, 1989).

Dans le bas-pays du nord de la France, deux événements rapides liés à des conditions hydrologiques marquées sont enregistrés au début du Néolithique final. Si les datations n'autorisent pas un calage temporel très précis et encore moins une mise en évidence d'une simultanéité, il est probable qu'ils interviennent tous deux aux environs de 4800 ans cal. BP (fig. 11).

Pendant qu'au Vignoble les taux de pollen d'hygrophytes dépassent les $20 \%$, le versant en pente forte en vis-à-vis (constitué de craie sur marne avec un contact lithologique induisant la présence de sources) subit un glissement de terrain (Valenciennes " rue Bernier », Collette in Deckers \& Delassus, 2009). L'événement est antérieur à une occupation néolithique datée d'environ 4000 à 4100 BP (date la plus ancienne sur os humain : $4110 \pm 40$ BP, Poz-22467). Il est apparemment isolé dans le temps et l'espace et est, dans tous les cas, lié à des conditions hydrologiques fortes.

La vallée de la Deûle, à Houplin-Ancoisne « Le Maraisde-Santes », enregistre une remontée de la nappe postérieure à $5210 \pm 40 \mathrm{BP}(\mathrm{GrA}-25616$, reprise d'un charbon de bois par l'érosion) et antérieure à $4165 \pm 20$ BP (GrN28442 , bois brûlé au sein du dépôt postérieur à l'érosion). Elle permet la réactivation de chenaux pléistocènes inscrits dans la morphologie d'une basse terrasse et s'accompagne d'une érosion de la berge (Houplin-Ancoisne, le Marais de Santes, Deschodt in Praud et al., sous presse). Légèrement plus en aval, à Houplin-Ancoisne "Station d'épuration », la stratigraphie, particulièrement homogène, du chenal holocène observé s'explique par un comblement entièrement postérieur à l'érosion. Il implique une vidange totale du chenal holocène dans certaines sections du lit mineur de la Deûle, suivie d'un nouveau comblement au Subboréal (réinterprétation des données de Deschodt et al., 2004).

L'enregistrement de Valenciennes « le Vignoble » montre que l'évolution vers des conditions ponctuellement plus humides précède les marqueurs locaux de néolithisation (fig. 11). Ceci n'exclut pas que l'anthropisation ait pu se conjuguer aux conditions climatiques plus humides à l'échelle du bassin et permettre le franchissement de seuil dans le fonctionnement du réseau hydrographique.

Dans le nord de la France, un schéma cohérent s'esquisse donc pour l'Atlantique (fig. 12) : une tendance générale à une humidité plus forte au cours de l'Atlantique aboutissant à une remontée de la nappe, à un changement de fonctionnement des vallées et à des phénomènes érosifs marqués à la fin de l'Atlantique. Toutefois les deux crises hydrologiques mises en évidence dans le bassin français de l'Escaut semblent intervenir tardivement par rapport aux indicateurs d'une dégradation climatique dans la deuxième partie du $5^{\mathrm{e}}$ millénaire (Bond et al., 2001 ; Berger, 2005).

\subsection{2 - Comparaison avec les bassins versants voisins}

Le détail de l'évolution et des relations entre l'enregistrement sédimentaire de fond de vallée et l'évolu- tion climatique est à rechercher à l'échelle régionale (Gronenborn, 2010 ; Macklin et al., 2010). Mais une grande prudence doit rester de mise sur des relations fondées sur la seule synchronicité (Houben et al., 2009 ; Notebaert \& Verstraeten, 2010). Peut-être ces événements paroxysmaux observés ne sont-ils que des accidents liés à une complexité et une variabilité climatique non perçue dans d'autres enregistrements locaux. Toutefois, l'hypothèse d'une évolution multiséculaire (humidité croissante, avec dans le détail des alternances de phases plus sèches) qui déclenche à terme ces phénomènes est privilégiée.

Plus à l'ouest, dans l'Audomarois, des assemblages entomologiques enregistrent un refroidissement (baisse des températures estivales) et une succession de phases humides et sèches entre environ 6700 et 3500 ans cal. BP (Gandouin et al., 2007). Cet enregistrement, plus tardif qu'au Vignoble, indique que l'alternance de ces phases, perçue dès environ 7900 ans cal. BP au Vignoble, se poursuit sur la fin de l'Atlantique et au Subboréal.

A une échelle un peu plus large, de nombreuses vallées du Bassin parisien ou de la Belgique montrent une évolution similaire, avec un arrêt ou un ralentissement de la sédimentation organique, l'incision de nouveaux chenaux et des apports détritiques plus abondants, parfois exclusifs. Le phénomène est commun à toutes les rivières européennes de latitude moyenne (Notebaert \& Verstraeten, 2010). Un recensement des données proches de Valenciennes (tab. 4) et le calage des fourchettes chronologiques des modifications (fig. 12) montrent qu'il n'est toutefois pas synchrone dans tous les enregistrements. Un décalage important peut exister entre les différents bassins versants mais aussi à l'intérieur d'un même bassin, parfois de faible superficie. Le cas est clairement mis en évidence dans le bassin de la Beuvronne, affluent de la Marne (Orth et al., 2004) ou dans de petits bassins affluents de la partie aval de l'Escaut comme la Dijle (Notebaert et al., 2011) et surtout la Mark (Huybrechts, 1989, 1999), où le colmatage argileux intervient précocement dès la première partie de l'Atlantique dans la section amont (datation vers 7600 BP, environ 8500 ans cal. BP). Dans la Somme, la tourbière de fond de vallée est ré-incisée entre 6000 et $5500 \mathrm{BP}$ (entre env. 6800 et 6300 ans cal. BP) (Antoine, 1997). Dans les bassins de la moyenne vallée de l'Oise, de la Marne aval, de l'Yonne aval et de la Seine en amont de Paris, les marqueurs (arrêt de la tourbogénèse, érosions, apports détritiques) s'étalent de la fin de l'Atlantique au début du Subboréal (Pastre et al., 1997, 2002, 2003 ; Orth et al., 2004). Les « crises » perçues dans le bassin amont de l'Escaut participent de cette évolution.

A l'échelle de l'Holocène, dans l'hémisphère nord, les éléments observés dans le bassin de l'Escaut s'inscrivent dans une tendance générale d'une dégradation climatique (plus frais, plus humide) liée au forçage orbital (Naughton et al., 2007 ; Magny et al., 2011, 2012). A une échelle plus restreinte, tous les auteurs accordent un rôle 


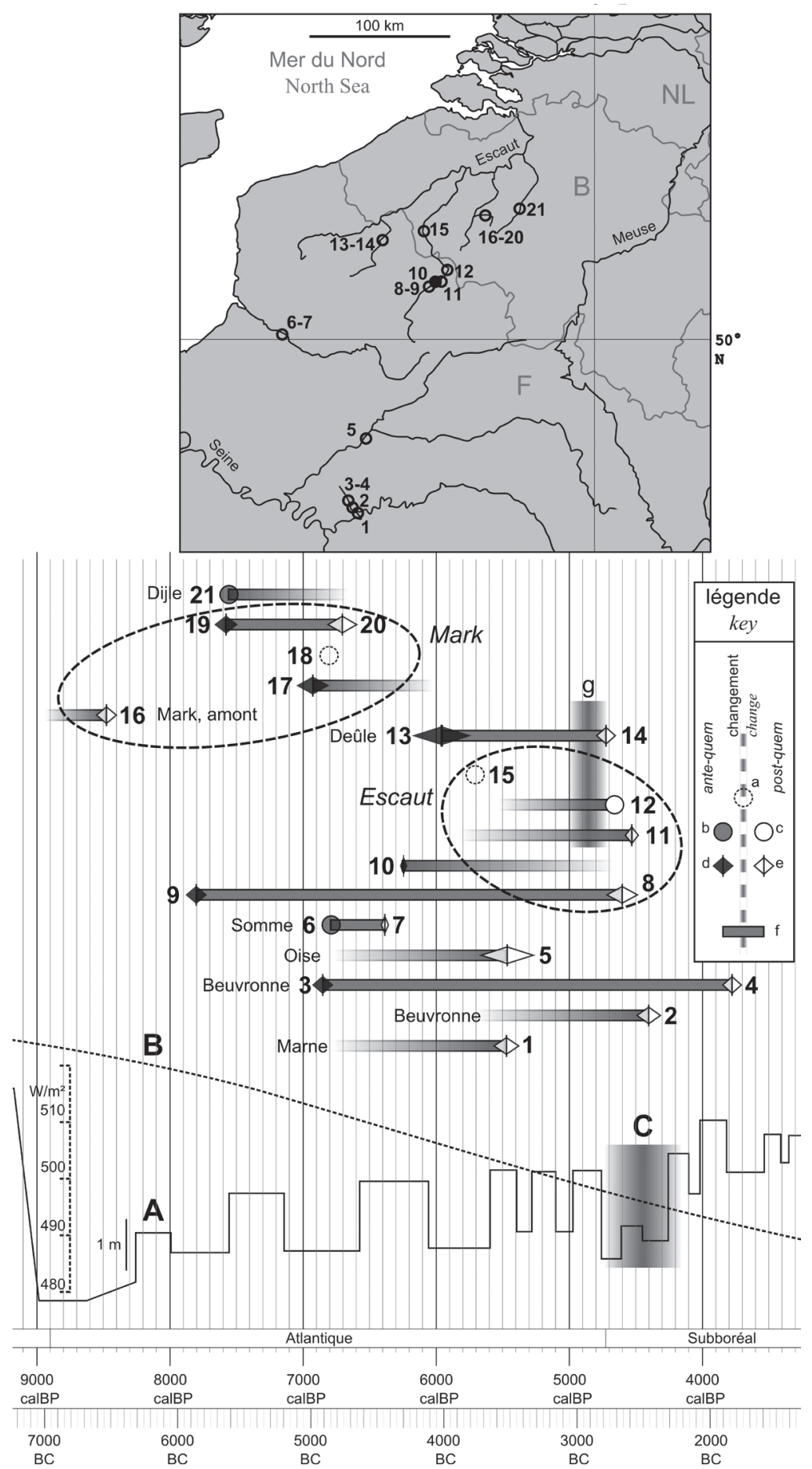

Fig. 12 : Chronologie des modifications des fonds de vallées tourbeux atlantique dans les bassins versants proches (détails et sources : tab. 4). a/ Datation approximative ou synthétique des modifications. b/ Datation approximative ou synthétique antérieure aux modifications. c/ Datation approximative ou synthétique postérieure aux modifications. $d$ / Datation radiocarbone antérieure aux modifications. e/ Datation radiocarbone postérieure aux modifications. f/ Intervalle chronologique des modifications. g/ Crise hydrologique probable dans le bassin amont de l'Escaut (fig. 11). A/ Variations du niveau du lac Cerin (Jura), Magny et al. (2011). B/ Insolation estivale à 65N, Berger (1978). C - phase de changements rapides, Magny et al. (2012).

Fig. 12: Chronology of changes in the Atlantic peaty valley bottoms in nearby catchments (details and sources: tab. 4). a/ Approximate or synthetic date of the changes. b/Approximate or synthetic date anterior to the changes. c/ Approximate or synthetic date posterior to the changes. d/ Radiocarbon date anterior to the changes. el Radiocarbon date posterior to the changes. f/ Chronological interval of the changes. $g /$ Probable hydrological crisis in the upstream Schelde catchment (fig. 11). A/ Variations of Cerin lake-level (Jura mountains), Magny et al. (2011). B/ Summer insolation at 65 ${ }^{\circ}$, Berger (1978). C/ Phase of rapid changes, Magny et al. (2012).

important à l'homme et à la néolithisation dans l'apport de sédiment détritique et les métamorphoses du système fluvial, souvent en association avec une dégradation climatique (Pastre et al., 1997, 2003 ; Orth et al., 2004 ; Chaussé et al., 2008). Le mode d'exploitation du sol est considéré comme prépondérant dans le cas de la Dijle (Notebaert et al., 2011), tandis que, dans le bassin voisin de la Mark, Huybrechts (1989) insiste sur une remontée de la nappe et le développement d'un plan d'eau en fond de vallée. 


\begin{tabular}{|c|c|c|c|c|c|c|c|c|c|c|}
\hline$n^{\circ}$ & source & $\begin{array}{l}\text { cours d'eau } \\
\text { river }\end{array}$ & $\begin{array}{l}\text { lieu } \\
\text { place }\end{array}$ & Latitude & Longit. & bass. v. & $\begin{array}{l}\text { unité datée } \\
\text { dated unit }\end{array}$ & date (BP) & $\begin{array}{l}\text { date cal. BP } \\
\text { post quem }\end{array}$ & $\begin{array}{l}\text { date } \\
\text { cal. BP } \\
\text { antequem }\end{array}$ \\
\hline 1 & Pastre et al. 1997 & Marne & Annet & 48,90 & 2,70 & grand & $\begin{array}{l}\text { chenal latéral incisant la tourbe } \\
\text { lateral channel in peat }\end{array}$ & $\begin{array}{l}4740+/-70 \\
\text { Gif } 8457\end{array}$ & - & $5463+/-99$ \\
\hline 2 & Orth et al. 2004 & Beuvronne & Claye-Souilly & 48,95 & 2,67 & petit & $\begin{array}{l}\text { début accumulation après incision tourbe } \\
\text { start infilling after peat incision }\end{array}$ & $\begin{array}{l}3950+/-60 \\
\text { Beta } 145190\end{array}$ & - & $4400+/-95$ \\
\hline 3 & Orth et al. 2004 & Biberonne & Compans & 48,98 & 2,67 & petit & $\begin{array}{l}\text { tourbe } \\
\text { peat }\end{array}$ & $\begin{array}{l}6000+/-60 \\
\text { Ly-1758(Oxa) }\end{array}$ & $6848+/-75$ & - \\
\hline 4 & Orth et al. 2004 & Biberonne & Compans & 48,98 & 2,67 & petit & $\begin{array}{l}\text { tourbe postérieure érosion } \\
\text { peat after erosion }\end{array}$ & $\begin{array}{l}3500+/-60 \\
\text { Beta } 142823\end{array}$ & to & $3781+/-73$ \\
\hline 5 & Pastre et al. 2002 & Oise & Armancourt & 49,37 & 2,77 & grand & $\begin{array}{l}\text { apport limoneux plus important } \\
\text { increase in silt input }\end{array}$ & $\begin{array}{l}4740+/-120 \\
\text { Gif } 11056\end{array}$ & - & $5458+/-125$ \\
\hline 6 & Antoine 1997 & Somme & environs d'Amiens & 49,90 & 2,30 & moyen & $\begin{array}{l}\text { fin développement tourbe } \\
\text { end of peat developpement }\end{array}$ & Env. 6000 & Env. 6800 & J \\
\hline 7 & Antoine 1997 & Somme & environs d'Amiens & 49,90 & 2,30 & moyen & $\begin{array}{l}\text { début accumulation après incision tourbe } \\
\text { start infilling after peat incision }\end{array}$ & $\begin{array}{l}5530+/-70 \\
\text { Gifa- } 9238\end{array}$ & Lan. & $4389+/-58$ \\
\hline 8 & perso, inédit & Escaut & Rouvignies-Haulchin & 50,33 & 3,44 & moyen & $\begin{array}{l}\text { activité chenal après incision tourbe } \\
\text { channel activity after peat incision }\end{array}$ & $\begin{array}{l}4064+/-49 \\
\text { Erl-17395 }\end{array}$ & - & $4598+/-121$ \\
\hline 9 & perso, inédit & Escaut & Rouvignies-Haulchin & 50,33 & 3,44 & moyen & $\begin{array}{l}\text { sommet de la tourbe } \\
\text { top of peat }\end{array}$ & $\begin{array}{l}6962+/-72 \\
\text { Erl-17394 } \\
\end{array}$ & $7806+/-82$ & 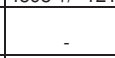 \\
\hline 10 & cet article & Escaut & Valenciennes & 50,35 & 3,50 & moyen & $\begin{array}{l}\text { sommet de la tourbe } \\
\text { top of peat }\end{array}$ & $\begin{array}{l}5422+/-43 \\
\text { Erl-11730 }\end{array}$ & $6240+/-37$ & - \\
\hline 11 & Deckers et Delassus 2009 & Escaut & Valenciennes & 50,35 & 3,55 & moyen & $\begin{array}{l}\text { première datation après glissement de terrain } \\
\text { first dating after landslide }\end{array}$ & $\begin{array}{l}4045+/-40 \\
\text { Ly-13989 }\end{array}$ & 然 & $4530+/-68$ \\
\hline 12 & Ruchard et al. 1992 & Escaut & aval Valenciennes & 50,40 & 3,60 & moyen & $\begin{array}{l}\text { alluvions limoneuses, datées par palynologie } \\
\text { silt alluvia, palynological dating }\end{array}$ & - & - & $\begin{array}{l}\text { env. } \\
4700 \text { cal BP }\end{array}$ \\
\hline 13 & Praud et al. 1997 & Deûle & Houplin-Ancoisne & 50,57 & 2,98 & petit & $\begin{array}{l}\text { charbon antérieure érosion } \\
\text { charcoal prior to erosion }\end{array}$ & $\begin{array}{l}5210+/-20 \\
\text { GrA-25616 }\end{array}$ & $5963+/-203$ & movardi \\
\hline 14 & Praud et al. 1997 & Deûle & Houplin-Ancoisne & 50,57 & 2,98 & petit & $\begin{array}{l}\text { travertin détritique après érosion } \\
\text { detritic calcareous tuffa after erosion }\end{array}$ & $\begin{array}{l}4165+/-20 \\
\text { GrN-28443 }\end{array}$ & $4729+1-72$ & - \\
\hline 15 & Kiden 1991 & Escaut & aval Tournai & 50,70 & 3,40 & moyen & $\begin{array}{l}\text { nouveaux chenaux incisés dans tourbe } \\
\text { new channels in peat }\end{array}$ & Env. 5000 & env. & 5700 \\
\hline 16 & Huybrechts 1989 & Mark & & 50,75 & 3,96 & petit & $\begin{array}{l}\text { base de l'unité argileuse sur tourbe, partie amont } \\
\text { clay unit bottom over peat, upwards reach }\end{array}$ & $\begin{array}{l}7620+/-90 \\
\text { IRPA } 552\end{array}$ & - & $8440+/-80$ \\
\hline 17 & Huybrechts 1989 & Mark & & 50,75 & 3,96 & petit & $\begin{array}{l}\text { sommet de la tourbe, partie aval } \\
\text { top of peat, downards reach }\end{array}$ & $\begin{array}{l}6050+/-75 \\
\text { IRPA } 570\end{array}$ & $6919+/-106$ & - \\
\hline 18 & Huybrechts 1989 & Mark & & 50,75 & 3,96 & petit & montée niveau nappe / water table rising & Env. $6000 \mathrm{BP}$ & env. & 6800 \\
\hline 19 & Huybrechts 1989 & Mark & & 50,75 & 3,96 & petit & sommet de la tourbe / top of peat & $\begin{array}{l}6710+/-100 \\
\text { IRPA } 440\end{array}$ & $7579+/-79$ & - \\
\hline 20 & Huybrechts 1989 & Mark & & 50,75 & 3,96 & petit & $\begin{array}{l}\text { base de l'unité argileuse sur tourbe } \\
\text { clay unit bottom over peat }\end{array}$ & $\begin{array}{l}5800+/-95 \\
\text { IRPA } 443\end{array}$ & - & $6609+/-109$ \\
\hline 21 & Notebaert et al. 2011 & Dijle & & 50,88 & 4,70 & petit & $\begin{array}{l}\text { début de l'arrêt sédimentation organique } \\
\text { organic sedimentation stop beginning }\end{array}$ & - & env. 7550 & - \\
\hline
\end{tabular}

Tab. 4 : Chronologie des modifications des fonds de vallées tourbeux atlantiques dans les bassins versants proches. Localisation : voir figure 13 ; courbe de calibration : CalPal2007 Hulu (Weninger \& Jöris, 2008).

Tab. 4: Chronology of changes in Atlantic peaty valley bottoms in nearby catchments. Location: see figure 13; calibration curve: CalPal2007 Hulu (Weninger \& Jöris, 2008).

\section{7 - CONCLUSION}

La partie étudiée du fond de vallée de l'Escaut est un pied de versant envahi par une tourbe au cours de l'Atlantique. La croissance de la tourbe ralentit et diminue fortement à partir d'environ 6000 ans cal. BP. Par la suite, la zone étudiée devient une zone humide morphologiquement stable, n'enregistrant qu'un long hiatus, sans trace d'érosion, jusqu'aux apports massifs de colluvions limoneuses médiévales et modernes.

La séquence fournit un enregistrement atlantique particulièrement dilaté $(1,5 \mathrm{~m})$ qui bénéficie d'un calage chronologique précis (sept dates). L'analyse palynologique témoigne d'un développement du tilleul localement particulièrement important dès l'Atlantique ancien. Ce ligneux est cependant sensible aux conditions édaphiques et présente ici des phases successives de recul. Celles-ci sont, pour la plupart, corrélées à des augmentations des indicateurs d'humidité, certes faiblement perçues, mais néanmoins enregistrées dans les courbes. Ces conditions plus humides se situent aux environs de 7950, 7750, 7550,7150 et 6900 ans cal. BP et après 6000 ans cal. BP (fig. 7 et 11). Elles culminent avec la transformation du fond de vallée en zone plus ouverte et marécageuse et, paradoxalement, avec la fin de la tourbification (ré-incision de chenaux qui répondrait à une augmentation du débit ?). Les premiers marqueurs de plus forte humidité sont antérieurs à la néolithisation du secteur.

Une corrélation est proposée avec, d'une part, la modification des écoulements enregistrés dans la vallée (fin de la sédimentation tourbeuse et travertineuse généralisée, incision de nouveaux chenaux, apports limoneux) et, d'autre part, des crises hydrologiques observées dans la vallée de l'Escaut (glissement de terrain) et de la Deûle (montée de la nappe, crise érosive) à la fin de l'Atlantique.

Le phénomène s'inscrit dans une tendance supra-régionale de réponse des vallées tourbeuses à une dégradation climatique (plus humide) sensible sur le long terme (plusieurs millénaires) mais irrégulière dans le détail. De courtes périodes d'extrêmes climatiques difficiles à mettre en évidence et surtout un déséquilibre induit par la néolithisation et son impact croissant (érosion des sols, apport sédimentaire, bilan évapotranspiration) ont pu faciliter le franchissement de seuils et provoquer une réponse non linéaire des cours d'eau. Les différents bassins et parfois, à l'intérieur d'un même bassin, les différentes sections d'un cours d'eau, réagissent de manière diachrone. Dans la région considérée, les premières modifications du système fluvial interviennent dès la première partie de l'Atlantique (bassin de la Mark). La plupart des fonds de vallées semblent être affectés au cours de la deuxième partie de l'Atlantique. Au début du Subboréal, le changement est généralisé.

\section{REMERCIEMENTS}

Nous remercions Gilles Leroy qui a prescrit l'étude, Pierre-Gil Salvador, Chantal Leroyer ainsi que Vincent Lebreton dont les remarques et les conseils ont permis de notables améliorations. 


\section{RÉFÉRENCES BIBLIOGRAPHIQUES}

ANTOINE P., 1997 - Modifications des systèmes fluviatiles à la transition Pléniglaciaire-Tardiglaciaire et à l'Holocène : l'exemple du bassin de la Somme (Nord de la France). Géographie Physique et Quaternaire, 51 (1), 93-106.

ANTOINE P., FAGNART J.-P., LIMONDIN-LOZOUET N. \& MUNAUT A.-V., 2000 - Le Tardiglaciaire du bassin de la Somme : éléments de synthèse et nouvelles données. Quaternaire, 11 (2), 85-98.

BEHRE K.-E., 1981 - The interpretation of anthropogenic indicators in pollen diagrams. Pollen et Spores, 23 (2), 225-245.

BERGER A., 1978 - Long-term variations of caloric insolation resulting from the earth's orbital elements. Quaternary Research, 9 (2), 139-167.

BERGER J.-F., 2005 - Sédiments, dynamique du peuplement et climat au Néolithique ancien. In J. Guilaine (ed.), Populations néolithiques et environnements. Collection des Hespérides. Editions Errance, Paris, 155-212.

BLAMEY M. \& GREY-WILSON C., 1991 - La flore d'Europe occidentale. Arthaud, Paris, 544 p.

BOND G., KROMER B., BEER J., MUSCHELER R., EVANS M., SHOWERS W., HOFFMANN S, LOTTI-BOND R, HAJDAS I. \& BONANI G, 2001 - Persistent Solar Influence on North Atlantic Climate During the Holocene. Science, 294 (5549), 2130-2136.

BONNIER G. \& DE LAYENS G., 2008 - Nouvelle flore du Nord de la France et de la Belgique pour la détermination facile des plantes. Belin, Paris, $310 \mathrm{p}$

BOREL J.-L., BROCHIER J.-L., COLARDELLE M. \& LUNDSTRÖM-BAUDAIS K., 1985 - Une expérience de recherche concertée sur le paléoenvironnement de l'habitat médiéval immergé de Colletière à Charavines-les-Bains (Isère). In J. Renault-Miskovsky, BuiThi-Mai \& M. Girard (eds.), Palynologie archéologique : actes des journées des 25-26-27 janvier 1984. Notes et Monographies Techniques - Centre de Recherches Archéologiques, 17. Editions du CNRS, Paris, 313-330.

BOULEN M., 1995 - Analyse pollinique de séquences tourbeuses. In P. Le Guen \& M. Boulen, Varesne "le Pré Hareng » (60), Courbes "le Pinteloret" (02), Courbes "les Pâtures » (02). Rapport d'évaluation, Service Régional d'Archéologie de Picardie, Amiens, 6-33.

BOULEN M., 1997 - Etudes palynologiques en contextes archéologique et naturel - La micro-aire de Bucy-le-Long (Aisne). Diplôme de l'EHESS, Ecole des Hautes Etudes en Sciences Sociales, Toulouse, $151 \mathrm{p}$

BOULEN M., 2001 - Analyse pollinique de séquences tourbeuses. In S. Gaudefroy \& M. Boulen, Courbes "les Prés Ménart» - "les Quarante Faulx " (02). Rapport d'évaluation, Association pour les Fouilles Archéologiques Nationales \& Service Régional d'Archéologie de Picardie, Amiens, 18-28.

BOULEN M., 2011a - Le paysage au Néolithique dans le nord de la France : nouvelles données sur le Subboréal. Revue Archéologique de Picardie. Numéro spécial, 28, 301-311.

BOULEN M., 2011b - Evolution de l'environnement sur la plaine de Soupir/Moussy-Verneuil (Aisne), d'après les analyses palynologiques de structures archéologiques et de paléochenaux. Mémoire de Master 2, Université Paris 1 Panthéon-Sorbonne, Paris, 94 p

BOURNÉRIAS M., 1968 - Guide des groupements végétaux de la région parisienne. SEDES, Paris, $287 \mathrm{p}$

BRUN C., 2007 - Archéophytes et néophytes. Pour une nouvelle détermination des marqueurs polliniques de l'anthropisation. Le cas des milieux cultivés et rudéraux en Franche-Comté. Thèse de Doctorat, Université de Franche-Comté, Besançon, 421 p.

BRUN C., 2009 - Biodiversity changes in highly anthropogenic environments (cultivated and rudéral) since the Neolithic in eastern France. The Holocene, 19 (6), 861-871.

CHAUSSÉ C., LEROYER C., GIRARDCLOS O., ALLENET G., PION P. \& RAYMOND P., 2008 - Holocene history of the River Seine, Paris, France: bio-chronostratigraphic and geomorphological evidence from the Quai-Branly. The Holocene, 18 (6), 967-980.

DECKERS M. \& DELASSUS D., 2009 - Valenciennes, Vallée de L'Escaut, un site du Néolithique final. Monographie, Service Archéologique de Valenciennes, Valenciennes, $167 \mathrm{p}$

DESCHODT L., 2001 - Contribution à l'étude du Quaternaire de la vallée de l'Escaut, région de Condé-sur-l'Escaut, dans le cadre du projet européen Planarch. Etude documentaire, Institut National de Recherches Archéologiques Préventives \& Service Régional de l'Archéologie du Nord-Pas-de-Calais, Lille, $316 \mathrm{p}$

DESCHODT L., 2002 - Une phase de sédimentation alluviale au Préboréal initial dans la vallée de l'Escaut (Nord de la France). Quaternaire, 13 (2), 149-152.
DESCHODT L., SALVADOR P.-G. \& BOULEN M., 2004 - Formations sédimentaires et évolution de la vallée de la Deûle depuis le Pléniglaciaire supérieur à Houplin-Ancoisne (Nord de la France). Quaternaire, 15 (3), 269-284.

DESCHODT L., BOULEN M., CERCY Ch. \& DESSAUX N., 2006 - Nouvelles données archéologiques sur la Deûle lilloise d'une crise érosive du $\mathrm{II}^{\mathrm{e}} \mathrm{s}$. ap. J.-C. à l'urbanisation du lit mineur. Revue du Nord, 88 (368), 9-31.

EMONTSPOHL A.F., 1994 - Enregistrement pollinique du début du Weichselien et de 1'Holocène à Fampoux, vallée de la Scarpe (Nord, France). Quaternaire, 5 (1), 35-40.

EMONTSPOHL A.-F. \& VERMEERSCH D., 1991 - Premier exemple d'une succession Bølling-Dryas II-Allerød en Picardie (Famechon, Somme). Quaternaire, 2 (1), 17-25.

FAEGR K. \& IVERSEN J., 1989 - Textbook of Pollen Analysis, $4^{\text {th }}$ edition. Blackburn Press, Caldwell, $328 \mathrm{p}$.

GANDOUIN E., PONEL P., FRANQUET E., VAN VLIET-LANOË B., ANDRIEU-PONEL V., KEEN D.H., BRULHET J. \& BROCANDEL M., 2007 - Chironomid responses (Insect: Diptera) to Younger Dryas and Holocene environmental changes in a river floodplain from northern France (St-Momelin, St-Omer basin). The Holocene, 17 (3), 331-347.

GAUDIN L., 2004 - Les transformations spatio-temporelles de la végétation du nord-ouest de la France depuis la fin de la dernière glaciation. Reconstitutions paléo-paysagères. Thèse de Doctorat, Université de Rennes 1, Rennes, 2 vol., 763 p.

GRONENBORN D, 2010 - Climate, crises, and the "Neolithisation " of Central Europe between IRD-Events 6 and 4. In D. Gronenborg \& J. Petrasch (dir.), Die Neolithisierung Mitteleuropas : internationale Tagung, Mainz, 24.-26. Juni 2005. Verlag des Römisch-Germanischen Zentralmuseums, Mainz, 61-80.

HAVINGA A.J., 1984 - A 20-year experimental investigation into the differential corrosion susceptibility of pollen and spores in various soil types. Pollen et Spores, 26 (3-4), 541-558.

HENTON A., BOULEN M. \& DESCHODT L., 2009 - Valenciennes (59). Le Vignoble $I V$. Rapport final d'opération, Service Régional d'Archéologie du Nord - Pas-de-Calais, Lille, 57 p.

HOUBEN P., WUNDERLICH J. \& SCHROTT L., 2009 - Climate and long-term human impact on sediment fluxes in watershed systems. Geomorphology, 108 (1-2), 1-7

HUYBRECHTS W., 1989 - Palaeohydrologic conditions in the Mark River basin during the last 15,000 years. Geologie en Mijnbouw, 68 (2), 175-187.

HUYBRECHTS W., 1999 - Post-pleniglacial floodplain sediments in Central Belgium. Geologica Belgica, 2 (3-4), 29-37.

KIDEN P., 1991 - The Lateglacial and Holocene evolution of the middle and lower River Scheldt, Belgium. In L. Starkel, K.J. Gregory \& J.B. Thornes (eds.), Temperate palaeohydrology. John Wiley \& Sons, Chichester, New York \& Brisbane, 283-299.

LERICHE B., 2009 - Rouvignies (59), Extension du parc d'activités de l'aérodrome ouest (plateau d'Hérin) : phase 13B. Rapport de diagnostic, Institut National de Recherches Archéologiques Préventives \& Service Régional de l'Archéologie de Nord-Pas-de-Calais, Lille, 29 p.

LEROYER C., 1997 - Homme, climat, végétation au tardi- et postglaciaire dans le Bassin parisien : apports de l'étude palynologique des fonds de vallée. Thèse de Doctorat, Université Paris 1 PanthéonSorbonne, $786 \mathrm{p}$.

LEROYER C., PASTRE J.-F., FONTUGNE M. \& LIMONDINLOZOUET N., 1997 - Le Tardiglaciaire et le début de l'Holocène dans le bassin aval de la Marne (Seine-et-Marne - France) : Chronostratigraphie et environnement des occupations humaines. In J.-P. Fagnart \& A. Thévenin (eds.), Le Tardiglaciaire en Europe du NordOuest, Actes du $119^{\mathrm{e}}$ Congrès national des sociétés historiques et scientifiques, Section de pré- et protohistoire, Amiens, 26-30 octobre 1994. Editions du CTHS, Paris, 151-164.

LEROYER C., BOULEN M., MARGUERIE D., LORHO T., PRAT B. \& ARGANT J., avec les contributions de ALLENET DE RIBEMONT G., AOUSTIN D., DIOT M.-F., GAUDIN L., GUENET P., LATOUR-ARGANT C., MAREMBAT L., PERRIÈRE J., TIXIER C. \& VIVENT D., 2009 - Base de données S.I.G. palynologiques sur l'Age du Fer en France : une autre approche du paysage végétal et de son anthropisation. In I. Bertrand, A. Duval, J. Gomez de Soto \& P. Maguer (dir.), Habitats et paysages ruraux en Gaule et regards sur d'autres régions du monde celtique. Actes du XXXI colloque international de l'Association Française pour l'Etude de l'Age du Fer, 17-20 mai 2007, Chauvigny (Vienne, F). Tome II. Mémoire - Société de recherches archéologiques de Chauvigny, 35. Association des Publications Chauvinoises, Chauvigny, 447-468 
LEROYER C., BOULEN M. \& AUBRY L., avec les contributions de AOUSTIN D., ALLENET DE RIBEMONT G. \& PERRIÈRE J., 2013 - Environnement et incidence des occupations du Néolithique moyen dans la sphère Michelsberg : apport de la base de données polliniques. In J.-P. Demoule \& F. Lüth (dir.), Emergence de la complexité sociale : enceintes, ressources stratégiques et territorialité au Néolithique. Recherches franco-allemandes sur la culture de Michelsberg. Rapport de projet ANR, Université Paris 1 Panthéon Sorbonne \& CNRS, Paris, 50 p.

LOCHT J.-L., 2005 - Bruay-sur-Escaut. Diagnostic archéologique. GA0213. Institut National de Recherches Archéologiques Préventives \& Service Régional de l'Archéologie de Nord-Pas-de-Calais, Lille, $21 \mathrm{p}$.

MACAIRE J.-J., FOURMONT A., ARGANT J., BRÉHÉRET J-G., HINSCHBERGER F. \& TRÉMENT F., 2010 - Quantitative analysis of climate $v s$ human impact on sediment yield since the Late Glacial: the Sarliève paleolake catchment (France). The Holocene, 20 (4), 497-516.

MACKLIN M., JONES A. \& LEWIN J., 2010 - River response to rapid Holocene environmental change: evidence and explanation in British catchments. Quaternary Science Reviews, 29 (13-14), 1555-1576.

MAGNY M., 1995 - Une histoire du climat : des derniers mammouths au siècle de l'automobile. Editions Errance, Paris, 175 p.

MAGNY M. BOSSUET G, RUFFALDI P, LEROUX A. \& MOUTHON J., 2011 - Orbital imprint on Holocene palaeohydrological variations in west-central Europe as reflected by lake-level changes at Cerin (Jura Mountains, eastern France). Journal of Quaternary Science, 26 (2), 171-177.

MAGNY M., PEYRON O., SADORI L., ORTU E., ZANCHETTA G., VANNIERE B. \& TINNER W., 2012 - Contrasting patterns of precipitation seasonality during the Holocene in the south- and north-central Mediterranean. Journal of Quaternary Science, 27 (3), 290-296.

MARGUERIE D., 2009 - Interactions sociétés-milieux dans le nordouest de la France à l'Holocène récent : méthodologie et données archéobotaniques. Mémoire d'Habilitation à Diriger des Recherches, Université Rennes 1, Rennes, 439 p.

MUNAUT A.-V., 1967 - Recherches paléo-écologiques en basse et moyenne Belgique. Acta Geographica Lovaniensia, 6. Thèse de Doctorat, Université de Louvain, Louvain, 191 p.

NAUGHTON F., BOURILLET F., SÁNCHEZ GOÑI M. F., TURON J.-L. \& JOUANNEAU J.-M., 2007 - Long-term and millennialscale climate variability in northwestern France during the last 8850 years. The Holocene, 17 (7), 939-953.

NOTEBAERT B. \& VERSTRAETEN G., 2010 - Sensitivity of West and Central European river systems to environmental changes during the Holocene: A review. Earth-Science Reviews, 103 (3-4), 163-182.

NOTEBAERT B., HOUTBRECHTS G., VERSTRAETEN G., BROOTHAERTS N., HAECKX J., REYNDERS M., GOVERS G., PETIT F. \& POESEN J., 2011 - Fluvial architecture of Belgian river systems in contrasting environments: implications for reconstructing the sedimentation history. Nethrlands Journal of Geoscience, 90 (1), 31-50.

ORTH P., PASTRE J.-F, GAUTHIER A., LIMONDIN-LOZOUET N. \& KUNESCH S., 2004 - Les enregistrements morphosédimentaires et biostratigraphiques des fonds de vallée du bassin-versant de la Beuvronne (Bassin parisien, Seine-et-Marne, France) : perception des changements climatoanthropiques à l'Holocène. Quaternaire, 15 (3), 285-298.
PASTRE J.-F., FONTUGNE M., KUZUCUOGLU C., LEROYER C., LIMONDIN-LOZOUET N., TALON M. \& TISNÉRATLABORDE N., 1997 - L'évolution tardi- et postglaciaire des lits fluviaux au nord-est de Paris (France). Relations avec les données paléoenvironnementales et l'impact anthropique sur les versants. Géomorphologie : Relief, Processus, Environnement, 3 (4), 291-312.

PASTRE J.-F, LEROYER C., LIMONDIN-LOZOUET N., ORTH P., CHAUSSÉ C., FONTUGNE M., GAUTHIER, A., KUNESCH S., LE JEUNE Y. \& SAAD M.-C., 2002 - Variations paléoenvironnementales et paléohydrologiques durant les 15 derniers millénaires : les réponses morpho-sédimentaires des vallées du Bassin parisien (France). In J.-P. Bravard \& M. Magny (dir.), Histoire des rivières et des lacs de Lascaux à nos jours. Editions Errance, Paris, 29-44.

PASTRE J.-F., LEROYER C., LIMONDIN-LOZOUET N., ANTOINE P., GAUTHIER A., LE JEUNE Y. \& ORTH P. 2003 - Quinze mille ans d'environnement dans le Bassin parisien (France) : mémoires sédimentaires des fonds de vallées. In $\mathrm{T}$. Muxart, F.-D. Vivien, B. Villalba \& J. Burnouf (eds.), Des milieux et des hommes : fragments d'histoires croisées. Elsevier, Paris, 43-55.

PRAUD I. (dir.), BERNARD V., BOITARD E., BOULEN M. BRAGUIER S., CAYOL N., COUBRAY S., DESCHODT L., FECHNER K., HAMON C., LANCELOT S., MAIGROT Y., MARTIAL E., MÉDARD F., MONCHABLON C., PONEL P. \& DIETSCH-SELLAMI M.-F., sous presse - Houplin-Ancoisne "Le Marais de Santes » : une implantation au bord de la Deûle au Néolithique final. Recherches archéologiques. INRAP \& CNRS, Paris.

RAMEAU J.-C., MANSION D. \& DUMÉ G., 1989 - Flore forestière française : guide écologique illustré. Tome 1, Plaines et collines Institut pour le Développement Forestier, Ministère de 1'Agriculture et de la Forêt, Direction de l'Espace Rural et de la Forêt \& Ecole Nationale du Génie Rural, des Eaux et des Forêts, Paris, 1785 p.

REILLE M., 1990 - Leçons de palynologie et d'analyse pollinique. Editions du CNRS, Paris, $206 \mathrm{p}$.

REIMER P.J., BAILLIE M.G.L., BARD E., BAYLISS A., BECK J.W., BERTRAND C.J.H., BLACKWELL P.G., BUCK C.E., BURR G.S., CUTLER K.B., DAMON P.E., EDWARDS R.L., FAIRBANKS R.G., FRIEDRICH M., GUILDERSON T.P., HOGG A.G., HUGHEN K.A., KROMER B., MCGORMAC G., MANNING S., BRONK RAMSEY C., REIMER R.W., REMMELE S., SOUTHON J.R., STUIVER M., TALAMO S., TAYLOR F.W., VAN DER PLICHT J. \& WEYHENMEYER C.E., 2004 - IntCal04 terrestrial radiocarbon age calibration, 0-26 cal kyr BP. Radiocarbon, 46 (3), 1029-1058.

RICHARD H., 1995 - Analyse de l'anthropisation du milieu à partir de quelques exemples de variations de pollens d'arbres et d'arbustes. In S.E. Van der Leeuw (ed.), L'Homme et la dégradation de l'environnement. Actes des XV Rencontres Internationales d'Archéologie et d'Histoire d'Antibes, 20-22 octobre 1994. Editions APDCA, Juanles-Pins, 143-159.

RUCHARD N., LENAERTS S. \& MUNAUT A.-V., 1992 - Etude palynologique de sédiments holocènes dans la plaine alluviale du Haut-Escaut (Nord, France). Les Cahiers de Préhistoire du Nord, 10 118-130.

VERBRUGGEN C. \& KIDEN p., 1989 - L'évolution postglaciaire du Bas et Moyen Escaut. Les Cahiers de Préhistoire du Nord, 6, 5-14

WENINGER B. \& JÖRIS O., 2008 - A ${ }^{14} \mathrm{C}$ age calibration curve for the last $60 \mathrm{ka}$ : the Greenland-Hulu U/Th timescale and its impact on understanding the Middle to Upper Paleolithic transition in Western Eurasia. Journal of Human Evolution, 55 (5), 772-781. 